\title{
Synthesis of some novel pyrimidine, thiophene, coumarin, pyridine and pyrrole derivatives and their biological evaluation as analgesic, antipyretic and anti-inflammatory agents
}

\author{
Karam Ahmed El-Sharkawy, ${ }^{1,2 *}$ Mohammed Mofreh AlBratty, ${ }^{1}$ Hassan Ahmad Alhazmi ${ }^{1}$ \\ ${ }^{1}$ Department of Pharmaceutical Chemistry, College of pharmacy, Jazan University, Jazan 45142, Saudi Arabia, ${ }^{2}$ Department of \\ Chemistry, Faculty of Biotechnology, October University for Modern Sciences and Arts(MSA), Egypt
}

\begin{abstract}
Pyrimidine derivative 3 was afforded through the reaction of compound (1) with 5-ureidohydantion (2). Product 3 underwent a cyclization to produce fused pyrimidine derivative 7, although the latter product 7 was synthesized through one step via the reaction of compound (1) with 5-ureidohydantion (2) using another catalyst. Compound $\mathbf{3}$ was oriented to react with cyclic ketones $\mathbf{8 a}, \mathbf{b}$ in the presence of elemental sulfur, salicylaldehyde (10), aryldiazonium chlorides 12a,b and $\omega$-bromo-4-methoxy- acetophenone (14), which afforded, fused thiophene derivatives $9 \mathbf{a}, \mathbf{b}$, coumarin derivative 11, arylhdrazono derivatives 13a,b and 4-methoxyphenyl butenyl derivative 15, respectively. The latter product $\mathbf{1 5}$ was reacted with either potassium cyanide (16a) or potassium thiocyanide (16b) to form cyano and thiocyano derivatives 17a,b, respectively. Compound 17a underwent further cyclization to afford pyridopyrimidine derivative 19. Compound $\mathbf{1 5}$ was reacted with either hydrazine (20a) or phenylhydrazine (20b) to produce hydrazo derivatives $\mathbf{2 1 a}, \mathbf{b}$ and these products were cyclize to produce pyrrole derivatives $\mathbf{2 3 a}, \mathbf{b}$. Finally, 5-ureidohydantion (2) was reacted with compounds $\mathbf{2 4 a}, \mathbf{b}, \mathbf{c}$ to afford pyrimidine derivatives $\mathbf{2 5 a}, \mathbf{b}, \mathbf{c}$. The structures of the synthesized compounds were confirmed using IR, ${ }^{1} \mathrm{H}$ NMR, ${ }^{13} \mathrm{C}$ NMR and mass spectrometry techniques. Compounds $\mathbf{1 1}$ and $\mathbf{1 9}$ have promising as analgesic and antipyretic activities.
\end{abstract}

Keywords: Pyrimidine derivative. Thiophene. Coumarin. Pyridine. Pyrrole. Analgesic. Antipyretic and anti-inflammatory agents.

\section{INTRODUCTION}

A series of studies was introduced to discover that hydantoin derivatives, important heterocyclic compounds, act as antioxidant agents (Gus'kov et al., 2004). Moreover, Imidazolidine-2,4 dione derivatives are specific biologically active compounds and act as antiproliferative agents (Reddy et al., 2010), hypoglycemic, aldose reductase inhibitor agents (Iqbal et al., 2015) and Bcl-2 inhibitors (Wang et al., 2015).

Pyridopyrimidine derivatives have a wide variety of biological properties, including antileishmanial (Agarwal et al., 2005) and antitubercular activities (Horvati et al., 2015; Rajesh et al., 2011). Additionally fused thiophene derivatives have antitumor activity (Dallemagne et al.,

\footnotetext{
*Correspondence: K. A. El-Sharkawy. Department of Pharmaceutical Chemistry, College of Pharmacy, Jazan University. P.O. Box 114, Jazan 45142, Saudi Arabia. Phone: 00201010698361,0966559686517 / Fax: 00238371543.

E-mail:karamsyn@yahoo.com
}

2003) and pyrimidine derivatives containing the coumarin moiety have analgesic and anti-pyretic effects (Keri et al., 2010). Hydrazono derivatives have shown anticancer activity (Sztanke, Rzymowska, Sztanke, 2013) and pyrrole derivatives have antibacterial activity (Padmavathi et al., 2011). In this article we aimed to improve and discover the analgesic, antipyretic and anti-inflammatory activities of synthesized compounds.

\section{MATERIAL AND METHODS}

\section{General procedures}

The melting points of the synthesized compounds were determined in open capillaries and are uncorrected. Elemental analyses were performed on a Yanaco CHNS Corder elemental analyzer (Japan). IR spectra were measured using $\mathrm{KBr}$ discs on a Pye Unicam SP-1000 spectrophotometer. ${ }^{1} \mathrm{H}$ NMR and ${ }^{13} \mathrm{C}$ NMR spectra were measured on a Varian EM 390-200 MHz instrument 
with $\mathrm{CD}_{3} \mathrm{SOCD}_{3}$ as the solvent using TMS as an internal standard material, the chemical shifts were expressed as $\delta$ ppm. Mass spectra were recorded on Kratos (75 eV) MS equipment (Germany).

General procedures for the synthesis of compound: 3-(4,6-diamino-1-(2,5-dioxoimidazolidin-4-yl)-2-oxo-1,2dihydropyrimidin-5-yl)-3-iminopropanenitrile (3)

$\beta$-amino- $\alpha, \gamma$-dicyanocrotononitrile (1) (3.96 g, $0.03 \mathrm{~mol})$ was added to a 5 -ureidohydantoin solution (2) (4.743 g, $0.03 \mathrm{~mol})$ in $100 \mathrm{~mL}$ of ethanol containing dimethylformamide $(5 \mathrm{~mL})$ and triethylamine $(1.0 \mathrm{~mL})$ as a catalyst. The reaction mixture was heated under reflux for $6 \mathrm{~h}$, cooled and poured onto ice containing a few drops of $\mathrm{HCl}$. Then, the formed solid product was filtered out.

Compound 3: Faint yellow crystals from ethanol, yield $54 \%$, $4.701 \mathrm{~g}$, m.p. $168-170{ }^{\circ} \mathrm{C}$. IR $(\mathrm{KBr}): \mathrm{v} / \mathrm{cm}^{-1}=$ $3438-3355\left(2 \mathrm{NH}_{2}, 3 \mathrm{NH}\right), 2883\left(\mathrm{CH}_{2}\right), 2765(\mathrm{CH}), 2223$ (CN), 1673, 1668, 1661 (3CO), $1648(\mathrm{C}=\mathrm{C}) .{ }^{1} \mathrm{H}$ NMR $\left(\mathrm{DMSO}_{6}\right) \delta=3.05-3.13\left(\mathrm{~s}, 2 \mathrm{H}, \mathrm{CH}_{2}\right), 4.87,5.12(2 \mathrm{~s}, 4 \mathrm{H}$, $\mathrm{D}_{2} \mathrm{O}$-exchangeable, $\left.2 \mathrm{NH}_{2}\right), 5.74(\mathrm{~s}, 1 \mathrm{H}$, imidazolidindione ring), 8.35, 9.22, $9.79\left(3 \mathrm{~s}, 3 \mathrm{H}, \mathrm{D}_{2} \mathrm{O}\right.$-exchangeable, $\left.3 \mathrm{NH}\right)$. ${ }^{13} \mathrm{C}$ NMR: $\delta=40.9\left(\mathrm{CH}_{2}\right), 62.1(\mathrm{CH}), 106.4(\mathrm{C}=\mathrm{NH})$, $116.9(\mathrm{CN}), 120.1,148.6,152.7$ (pyrimidine $\mathrm{C}$ ), 164.4, 168.3, $171.7(3 \mathrm{C}=\mathrm{O})$. MS (relative intensity) $\mathrm{m} / \mathrm{z}: 290$ $\left(\mathrm{M}^{+}, 32.2 \%\right)$. Calcd for $\mathrm{C}_{10} \mathrm{H}_{10} \mathrm{~N}_{8} \mathrm{O}_{3}$ (290.24): C, 41.38; H, 3.47; N, 38.61\%. Found: C, 41.65; H, 3.24; N, 38.90\%.

General procedure for the synthesis of compound: 5-(4,5,7-triamino-2-oxopyrido-[2,3-d]pyrimidin-3(2H)-yl) imidazolidine-2,4-dione (7)

Method (A): A solution of compound 3 ( $0.58 \mathrm{~g}$, $0.002 \mathrm{~mol})$ in ethanol $(50 \mathrm{~mL})$ containing a catalytic amount of piperidine $(0.5 \mathrm{~mL})$ was heated under reflux for $5 \mathrm{~h}$, poured onto an ice/water mixture containing a few drops of hydrochloric acid. The formed solid product was collected by filtration.

Method (B): $\beta$-Amino- $\alpha, \gamma$-dicyanocrotononitrile (1) $(0.396 \mathrm{~g}, 0.003 \mathrm{~mol})$ was added to a solution of compound $2(0.474 \mathrm{~g}, 0.003 \mathrm{~mol})$ in sodium ethoxide $(0.003 \mathrm{~mol})$ [prepared by dissolving sodium metal $(0.069 \mathrm{~g}, 0.003$ $\mathrm{mol})$ in absolute ethanol $(50 \mathrm{~mL})]$. The reaction mixture was heated under reflux for $6 \mathrm{~h}$ and then evaporated under vacuum. The product was triturated with ethanol and the formed solid product was collected by filtration.

Compound 7: Brown crystals from ethanol, yield $66 \%, 0.575$ g, m.p. $115-117^{\circ} \mathrm{C}$. IR (KBr): v/ $\mathrm{cm}^{-1}=$ 3488-3327 (3NH, $2 \mathrm{NH}), 2783(\mathrm{CH}), 1695,1684,1662$ $(3 \mathrm{CO}), 1651(\mathrm{C}=\mathrm{N}) .{ }^{1} \mathrm{H}$ NMR $\left(\mathrm{DMSO}_{-} \mathrm{d}_{6}\right) \delta=4.78$,
4.93, 5.27 (3s, 6H, $\mathrm{D}_{2} \mathrm{O}$-exchangeable, $3 \mathrm{NH}_{2}$ ), 5.67 (s, $1 \mathrm{H}$, imidazolidindione ring), $6.87(\mathrm{~s}, 1 \mathrm{H}$, pyridine ring), 8.73, 9.95 (2s, 2H, $\mathrm{D}_{2} \mathrm{O}$-exchangeable, $\left.2 \mathrm{NH}\right) .{ }^{13} \mathrm{C}$ NMR: $\delta=55.7(\mathrm{CH}), 124.3,133.4,138.5,144.7,150.3,152.4$ (pyridine $\mathrm{C}$, pyrimidine $\mathrm{C}), 166.1,169.7,173.2(3 \mathrm{C}=\mathrm{O})$. Calcd for $\mathrm{C}_{10} \mathrm{H}_{10} \mathrm{~N}_{8} \mathrm{O}_{3}(290.24)$ : C, 41.38; H, 3.47; N, $38.61 \%$. Found: C, 41.11; H, 3.73; N, 38.99\%.

General procedure for the synthesis of compounds: 5-(4,6-diamino-5-((2-amino-5,6-dihydro-4Hcyclopenta[b]thiophen-3-yl)(imino)methyl)-2-oxopyrimidin-1(2H)-yl) imidazolidine-2,4-dione (9a) and 5-(4,6-diamino-5-((2-amino-4,5,6,7-tetrahydrobenzo [b]thiophen-3-yl(imino) methyl)-2-oxopyrimidin-1(2H) yl) imidazolidine-2,4-dione (9b)

To a solution of compound 3 ( $0.58 \mathrm{~g}, 0.002 \mathrm{~mol})$ in ethanol $(50 \mathrm{~mL})$ containing trimethylamine $(0.5 \mathrm{~mL})$, either cyclopentanone (8a) ( $0.168 \mathrm{~g}, 0.002 \mathrm{~mol})$ or cyclohexanone (8b) $(0.196 \mathrm{~g}, 0.002 \mathrm{~mol})$ together with elemental sulfur $(0.064 \mathrm{~g}, 0.002 \mathrm{~mol})$ were added. The reaction mixture was heated under reflux for $4 \mathrm{~h}$, cooled and poured onto an ice/ water mixture containing a few drops of $\mathrm{HCl}$. The formed precipitate was collected by filtration.

Compound 9a: Brown crystals from 1,4-dioxane, yield $63 \%, 0.489$ g, m.p. $202-204{ }^{\circ} \mathrm{C}$. IR $(\mathrm{KBr}): \mathrm{v} / \mathrm{cm}^{-1}=$ $3458-3336\left(3 \mathrm{NH}_{2}, 3 \mathrm{NH}\right), 2880\left(\mathrm{CH}_{2}\right), 2796(\mathrm{CH}), 1683$, 1674, $1663(3 \mathrm{CO}), 1653(\mathrm{C}=\mathrm{N}), 1646(\mathrm{C}=\mathrm{C}), 684(\mathrm{C}-\mathrm{S})$. ${ }^{1} \mathrm{H}$ NMR $\left(\mathrm{DMSO}_{6} \mathrm{~d}_{6}\right) \delta=2.07-2.18\left(\mathrm{~m}, 6 \mathrm{H}, 3 \mathrm{CH}_{2}\right), 4.54$, 4.65, $5.81\left(3 \mathrm{~s}, 6 \mathrm{H}, \mathrm{D}_{2} \mathrm{O}\right.$-exchangeable, $\left.3 \mathrm{NH}_{2}\right), 5.98(\mathrm{~s}, 1 \mathrm{H}$, imidazolidindione ring), 8.52, 8.77, $9.12\left(3 \mathrm{~s}, 3 \mathrm{H}, \mathrm{D}_{2} \mathrm{O}-\right.$ exchangeable, $3 \mathrm{NH}) .{ }^{13} \mathrm{C}$ NMR: $\delta=22.4,27.3,29.9$ (3 $\left.\mathrm{CH}_{2}\right), 51.3(\mathrm{CH}), 93.8(\mathrm{C}=\mathrm{NH}), 126.2,131.5,136.3,140.5$, 142.7, 149.1, 151.6 (thiophene $\mathrm{C}$, pyrimidine $\mathrm{C}$ ), 165.4, 168.8, $172.1(3 \mathrm{C}=\mathrm{O})$. Calcd for $\mathrm{C}_{15} \mathrm{H}_{16} \mathrm{~N}_{8} \mathrm{O}_{3} \mathrm{~S}(388.40): \mathrm{C}$, 46.38; H, 4.15; N, 28.85; S, 8.26\%. Found: C, 46.17; H, 3.89 ; N, 28.66; S, 8.01\%.

Compound 9b: Brown crystals from 1,4-dioxane, yield $58 \%, 0.477$ g, m.p. $212-214^{\circ} \mathrm{C}$. IR $(\mathrm{KBr}): \mathrm{v} / \mathrm{cm}^{-1}=$ $3460-3349\left(3 \mathrm{NH}_{2}, 3 \mathrm{NH}\right), 2882\left(\mathrm{CH}_{2}\right), 2768(\mathrm{CH}), 1677$, 1671, $1664(3 \mathrm{CO}), 1655(\mathrm{C}=\mathrm{N}), 1649(\mathrm{C}=\mathrm{C}), 676(\mathrm{C}-\mathrm{S})$. ${ }^{1} \mathrm{H} \mathrm{NMR}\left(\mathrm{DMSO}_{6} \mathrm{~d}_{6}\right) \delta=2.18-2.39\left(\mathrm{~m}, 8 \mathrm{H}, 4 \mathrm{CH}_{2}\right), 4.48$, $4.69,5.45\left(3 \mathrm{~s}, 6 \mathrm{H}, \mathrm{D}_{2} \mathrm{O}\right.$-exchangeable, $\left.3 \mathrm{NH}_{2}\right), 5.86(\mathrm{~s}, 1 \mathrm{H}$, imidazolidindione ring), 8.61, 8.86, $9.08\left(3 \mathrm{~s}, 3 \mathrm{H}, \mathrm{D}_{2} \mathrm{O}-\right.$ exchangeable, $3 \mathrm{NH}) .{ }^{13} \mathrm{C} \mathrm{NMR}: \delta=22.8,25.4,27.3,29.6$ $\left(4 \mathrm{CH}_{2}\right), 54.7(\mathrm{CH}), 97.1(\mathrm{C}=\mathrm{NH}), 128.1,134.7,138.6$, $141.5,146.6,150.8,153.3$ (thiophene $\mathrm{C}$, pyrimidine C), 162.2, 166.7, $170.6(3 \mathrm{C}=\mathrm{O})$. Calcd for $\mathrm{C}_{16} \mathrm{H}_{18} \mathrm{~N}_{8} \mathrm{O}_{3} \mathrm{~S}$ (402.43): C, 47.75; H, 4.51; N, 27.84; S, 7.97\%. Found: C, 47.56; H, 4.78; N, 28.09; S, 7.68\%. 
General procedure for the synthesis of compound:5-(4,6-diamino-5-(imino(2-oxo-2H-chromen-3-yl) methyl)-2-oxopyrimidin-1(2H)-yl)imidazolidine-2,4-dione (11)

Salicylaldehyde (10) (0.244 g, $0.002 \mathrm{~mol})$ was added to a solution of compound $\mathbf{3}(0.58 \mathrm{~g}, 0.002 \mathrm{~mol})$ in 1,4-dioxane $(50 \mathrm{~mL})$ containing piperidine $(0.5 \mathrm{~mL})$. The reaction mixture was heated under reflux for $5 \mathrm{~h}$ and then evaporated under vacuum. The solid product was triturated with ethanol and the formed solid product was collected by filtration.

Compound 11: Yellowish brown crystals from 1,4-dioxane, yield $70 \%, 0.553$ g, m.p. $185-187^{\circ} \mathrm{C}$. IR (KBr): $\mathrm{v} / \mathrm{cm}^{-1}=3443-3311\left(2 \mathrm{NH}_{2}, 3 \mathrm{NH}\right), 3052$ (CH-aromatic.), $2762(\mathrm{CH}), 1835,1681,1666,1660$ $(4 \mathrm{C}=\mathrm{O}), 1652(\mathrm{C}=\mathrm{N}), 1112(\mathrm{CO}), 1649(\mathrm{C}=\mathrm{C}) .{ }^{1} \mathrm{H}$ NMR $\left(\mathrm{DMSO}_{6} \mathrm{~d}_{6}\right) \delta=4.76,5.25\left(2 \mathrm{~s}, 4 \mathrm{H}, \mathrm{D}_{2} \mathrm{O}\right.$-exchangeable, $\left.2 \mathrm{NH}_{2}\right), 5.53(\mathrm{~s}, 1 \mathrm{H}$, imidazolidindione ring $), 6.73(\mathrm{~s}, 1 \mathrm{H}$, coumarin $\mathrm{H}-4)$, 7.51- $7.66\left(\mathrm{~m}, 4 \mathrm{H}, \mathrm{C}_{6} \mathrm{H}_{4}\right), 8.88,9.28,9.55$ $\left(3 \mathrm{~s}, 3 \mathrm{H}, \mathrm{D}_{2} \mathrm{O}\right.$-exchangeable, $\left.3 \mathrm{NH}\right) .{ }^{13} \mathrm{C}$ NMR: $\delta=59.5$ $(\mathrm{CH}), 95.3(\mathrm{C}=\mathrm{NH}), 125.5,131.9,136.8,140.7,144.5$, 147.2, 149.1, 151.3, 153.5 (pyrimidine $C$, coumarin $C$ ), 161.5, 165.8, 170.3, $181.5(4 \mathrm{C}=\mathrm{O})$. Calcd for $\mathrm{C}_{17} \mathrm{H}_{13} \mathrm{~N}_{7} \mathrm{O}_{5}$ (395.33): C, 51.65; H, 3.31; N, 24.80\%. Found: C, 51.93; $\mathrm{H}, 3.59 ; \mathrm{N}, 24.55 \%$.

General procedure for the synthesis of compounds:2-(4,6-diamino-1-(2,5-dioxo- imidazolidin-4-yl)-2oxo 1,2-dihydropyrimidin-5-yl)-2-imino- $N^{\prime}$-phenylacetohydrazonoyl cyanide (13a) and $\mathrm{N}^{\prime}$-(4-chlorophenyl)2-(4,6-diamino-1-(2,5-dioxo- imidazolidin-4-yl-2-oxo1,2-dihydropyrimidin-5-yl)-2-iminoaceto-hydrazonoyl cyanide (13b)

To a cold solution $\left(0-5^{\circ} \mathrm{C}\right)$ of pyrimidine derivative 3 $(0.58 \mathrm{~g}, 0.002 \mathrm{~mol})$ in ethanol $(50 \mathrm{~mL})$ containing sodium acetate $(0.164 \mathrm{~g}, 0.002 \mathrm{~mol})$ either benzenediazonium chloride (12a) or 4-chlorobenzenediazonium chloride (12b) $(0.002 \mathrm{~mol})$ [prepared by adding an aqueous sodium nitrite solution $(0.138 \mathrm{~g}, 0.002 \mathrm{~mol})$ to a cold solution of either aniline or 4-chloroaniline $(0.002 \mathrm{~mol})$ in the appropriate amount of glacial acetic acid at $\left(0-5^{\circ} \mathrm{C}\right)$ with continuous stirring] was added with continuous stirring. The reaction mixture was stirred at room temperature for an additional $4 \mathrm{~h}$ and the solid product was collected by filtration.

Compound 13a: Pale brown crystals from ethanol, yield $67 \%, 0.528$ g, m.p. $137-139{ }^{\circ} \mathrm{C}$. IR (KBr): $\mathrm{v} / \mathrm{cm}^{-1}=3476-3354\left(2 \mathrm{NH}_{2}, 4 \mathrm{NH}\right), 3053(\mathrm{CH}$ aromatic $)$, $2761(\mathrm{CH}), 2223(\mathrm{CN}), 1678,1667,1663$ (3CO), 1657
$(\mathrm{C}=\mathrm{N}), 1646(\mathrm{C}=\mathrm{C}) .{ }^{1} \mathrm{H}$ NMR $\left(\mathrm{DMSO}_{-} \mathrm{d}_{6}\right) \delta=4.54$, $5.17\left(2 \mathrm{~s}, 4 \mathrm{H}, \mathrm{D}_{2} \mathrm{O}\right.$-exchangeable, $\left.2 \mathrm{NH}_{2}\right), 5.61(\mathrm{~s}, 1 \mathrm{H}$, imidazolidindione ring), 7.31- $7.62\left(\mathrm{~m}, 5 \mathrm{H}, \mathrm{C}_{6} \mathrm{H}_{5}\right), 8.76$, 9.13, 9.38, $9.59\left(4 \mathrm{~s}, 4 \mathrm{H}, \mathrm{D}_{2} \mathrm{O}\right.$-exchangeable, $\left.4 \mathrm{NH}\right)$. ${ }^{13} \mathrm{C}$ NMR: $\delta=67.3(\mathrm{CH}), 92.4,109.2(2 \mathrm{C}=\mathrm{N}), 120.4$ (CN), 123.3, 125.9, 127.9, 130.4, 132.8, 134.6, 138.4, 142.5 (pyrimidine $\left.\mathrm{C}, \mathrm{C}_{6} \mathrm{H}_{5}\right), 163.9,166.8,169.3(3 \mathrm{C}=\mathrm{O})$. MS (relative intensity) $\mathrm{m} / \mathrm{z}: 394\left(\mathrm{M}^{+}, 17.9 \%\right)$. Calcd for $\mathrm{C}_{16} \mathrm{H}_{14} \mathrm{~N}_{10} \mathrm{O}_{3}$ (394.35): C, 48.73; H, 3.58; N, 35.52\%. Found: C, 48.48; H, 3.29; N, 35.80\%.

Compound 13b: Dark brown crystals from ethanol, yield $61 \%, 0.523$ g, m.p. $193-195^{\circ} \mathrm{C}$. IR (KBr): v/cm ${ }^{-1}$ $=3451-3290\left(2 \mathrm{NH}_{2}, 4 \mathrm{NH}\right), 3050(\mathrm{CH}$ aromatic $), 2770$ $(\mathrm{CH}), 2224(\mathrm{CN}), 1673,1663,1659(3 \mathrm{CO}), 1652(\mathrm{C}=\mathrm{N})$, $1647(\mathrm{C}=\mathrm{C}) .{ }^{1} \mathrm{H}$ NMR $\left(\mathrm{DMSO}_{-} \mathrm{d}_{6}\right) \delta=4.63,5.08(2 \mathrm{~s}, 4 \mathrm{H}$, $\mathrm{D}_{2} \mathrm{O}$-exchangeable, $\left.2 \mathrm{NH}_{2}\right), 5.89(\mathrm{~s}, 1 \mathrm{H}$, imidazolidindione ring), 7.44- 7.58 (d.d, $\left.4 \mathrm{H}, \mathrm{C}_{6} \mathrm{H}_{4}\right), 8.58,9.24,9.55,9.67(4 \mathrm{~s}$, $4 \mathrm{H}, \mathrm{D}_{2} \mathrm{O}$-exchangeable, $\left.4 \mathrm{NH}\right) .{ }^{13} \mathrm{C}$ NMR: $\delta=62.5(\mathrm{CH})$, 98.4, 106.4 $(2 \mathrm{C}=\mathrm{N}), 121.7(\mathrm{CN}), 122.5,124.8,125.8,128.3$, 131.6, 135.3, 137.7, 141.5 (pyrimidine $\mathrm{C}, \mathrm{C}_{6} \mathrm{H}_{5}$ ), 162.8, 165.7, $168.8(3 \mathrm{C}=\mathrm{O})$. MS (relative intensity) $\mathrm{m} / \mathrm{z}: 428$ $\left(\mathrm{M}^{+}, 23.4 \%\right)$. Calcd for $\mathrm{C}_{16} \mathrm{H}_{13} \mathrm{ClN}_{10} \mathrm{O}_{3}$ (428.79): C, 44.82; H, 3.06; N, 32.67\%. Found: C, 44.56; H, 3.33; N, 32.40\%.

General procedure for synthesis of compound: 4-bromo2-((4,6-diamino-1-(2,5-dioxo- imidazolidin-4-yl)-2-oxo1,2-dihydropyrimidin-5-yl) (imino) methyl)-3-(4-methoxyphenyl) but-2-enenitrile (15)

$\omega$-Bromo-4-methoxyacetophenone (14) $(0.524 \mathrm{~g}$, $0.002 \mathrm{~mol})$ was added to a solution of compound $\mathbf{3}(0.58 \mathrm{~g}$, $0.002 \mathrm{~mol})$ in 1,4-dioxane $(40 \mathrm{~mL})$. The reaction mixture was stirred at room temperature for $2 \mathrm{~h}$ and then poured on to a beaker containing ice/water mixture. The formed solid product was collected by filtration.

Compound 15: Brown crystals from ethanol, yield $72 \%, 0.712$ g, m.p. $121-123{ }^{\circ} \mathrm{C}$. IR (KBr): v $/ \mathrm{cm}^{-1}=3422$ $3286\left(2 \mathrm{NH}_{2}, 3 \mathrm{NH}\right), 3055(\mathrm{CH}$ aromatic $), 2986\left(\mathrm{CH}_{3}\right)$, $2867\left(\mathrm{CH}_{2}\right), 2766(\mathrm{CH}), 2225(\mathrm{CN}), 1680,1669,1662$ (3CO), $1658(\mathrm{C}=\mathrm{N}), 1648(\mathrm{C}=\mathrm{C}) .{ }^{1} \mathrm{H}$ NMR $\left(\mathrm{DMSO}_{6} \mathrm{~d}_{6}\right) \delta$ $=3.32\left(\mathrm{~s}, 3 \mathrm{H}, \mathrm{OCH}_{3}\right), 3.77\left(\mathrm{~s}, 2 \mathrm{H}, \mathrm{CH}_{2}\right), 4.81,5.29(2 \mathrm{~s}, 4 \mathrm{H}$, $\mathrm{D}_{2} \mathrm{O}$-exchangeable, $\left.2 \mathrm{NH}_{2}\right), 5.55(\mathrm{~s}, 1 \mathrm{H}$, imidazolidindione ring), 7.48-7.71 (d.d, $\left.4 \mathrm{H}, \mathrm{C}_{6} \mathrm{H}_{4}\right), 8.66,9.22,9.45(3 \mathrm{~s}, 3 \mathrm{H}$, $\mathrm{D}_{2} \mathrm{O}$-exchangeable, $\left.3 \mathrm{NH}\right) .{ }^{13} \mathrm{C}$ NMR: $\delta=41,2\left(\mathrm{CH}_{3}\right)$, $49.5\left(\mathrm{CH}_{2}\right), 65.3(\mathrm{CH}), 81.3,88.6(\mathrm{C}=\mathrm{C}), 103.7(\mathrm{C}=\mathrm{N})$, $118.3(\mathrm{CN}), 124.6,126.7,129.3,131.5,133.9,135.4$, 137.8 (pyrimidine $\left.\mathrm{C}, \mathrm{C}_{6} \mathrm{H}_{4}\right), 160.7,165.7,169.8(3 \mathrm{C}=\mathrm{O})$. MS (relative intensity) $\mathrm{m} / \mathrm{z}: 500\left(\mathrm{M}^{+}, 13.8 \%\right), 502\left(\mathrm{M}^{+}\right.$, 13.4\%), Calcd for $\mathrm{C}_{19} \mathrm{H}_{17} \mathrm{BrN}_{8} \mathrm{O}_{4}$ (501.29): C, 45.52; H, 3.42 ; N, 22.35\%. Found: C, 45.81; H, 3.19; N, 22.63\%. 
General procedure for the synthesis of compounds: 2-((4,6-diamino-1-(2,5-dioxo- imidazolidin-4-yl)-2oxo-1,2-dihydropyrimidin-5-yl) (imino) methyl)-3(4- methoxy-phenyl-pent-2-enedinitrile (17a) and 2-((4,6-diamino-1-(2,5-dioxo-imidazolidin-4-yl)-2oxo-1,2-dihydropyrimidin-5-yl)(imino)methyl)-3-(4methoxyphenyl)-4-thio-cyanatobut-2-enenitrile (17b)

Either potassium cyanide (16a) $(0.122$ g, 0.002 $\mathrm{mol})$ or potassium thiocyanate (16b) $(0.189 \mathrm{~g}, 0.002$ mol) was added to a solution of compound $\mathbf{1 5}$ (1.002 $\mathrm{g}, 0.002 \mathrm{~mol})$ in ethanol $(50 \mathrm{~mL})$ in water bath at 60 ${ }^{\circ} \mathrm{C}$, with continuous stirring. The reaction mixture was maintained in the water bath for $1 \mathrm{~h}$ at $60{ }^{\circ} \mathrm{C}$ and then poured into a beaker containing an ice/water mixture and a few drops of $\mathrm{HCl}$. The formed solid product was collected by filtration.

Compound 17a: Dark brown crystals from ethanol, yield $69 \%, 0.617$ g, m.p. $157-159^{\circ} \mathrm{C}$. IR (KBr): v/cm ${ }^{-1}$ $=3445-3266\left(2 \mathrm{NH}_{2}, 3 \mathrm{NH}\right), 3051(\mathrm{CH}$ aromatic $), 2978$ $\left(\mathrm{CH}_{3}\right), 2881\left(\mathrm{CH}_{2}\right), 2754(\mathrm{CH}), 2225,2223(2 \mathrm{CN}), 1682$, 1673, $1661(3 \mathrm{CO}), 1657(\mathrm{C}=\mathrm{N}), 1649(\mathrm{C}=\mathrm{C}) .{ }^{1} \mathrm{H}$ NMR $\left(\mathrm{DMSO}_{6}\right) \delta=3.41\left(\mathrm{~s}, 3 \mathrm{H}, \mathrm{OCH}_{3}\right), 3.76\left(\mathrm{~s}, 2 \mathrm{H}, \mathrm{CH}_{2}\right)$, $4.55,4.87\left(2 \mathrm{~s}, 4 \mathrm{H}, \mathrm{D}_{2} \mathrm{O}\right.$-exchangeable, $\left.2 \mathrm{NH}_{2}\right), 5.63(\mathrm{~s}$, $1 \mathrm{H}$, imidazolidindione ring), 7.33-7.54 (d.d, $4 \mathrm{H}, \mathrm{C}_{6} \mathrm{H}_{4}$ ), $8.45,8.76,9.33\left(3 \mathrm{~s}, 3 \mathrm{H}, \mathrm{D}_{2} \mathrm{O}\right.$-exchangeable, $\left.3 \mathrm{NH}\right) .{ }^{13} \mathrm{C}$ NMR: $\delta=38.1\left(\mathrm{CH}_{3}\right), 48.9\left(\mathrm{CH}_{2}\right), 62.7(\mathrm{CH}), 77.7,83.5$ $(\mathrm{C}=\mathrm{C}), 97.6(\mathrm{C}=\mathrm{N}), 116.5,119.2(2 \mathrm{CN}), 122.8,125.4$, 128.6, 130.6, 134.4, 136.7, 138.9 (pyrimidine $\mathrm{C}, \mathrm{C}_{6} \mathrm{H}_{4}$ ), $161.4,164.5,168.1(3 \mathrm{C}=\mathrm{O})$. MS (relative intensity) $\mathrm{m} / \mathrm{z}$ : $447\left(\mathrm{M}^{+}, 28.4 \%\right)$. Calcd for $\mathrm{C}_{20} \mathrm{H}_{17} \mathrm{~N}_{9} \mathrm{O}_{4}(447.41)$ : C, 53.69; H, 3.83; N, 28.18\%. Found: C, 53.96; H, 3.59; N, $28.43 \%$.

Compound 17b: Brown crystals from ethanol, yield $64 \%, 0.613$ g, m.p. $181-183^{\circ} \mathrm{C}$. IR (KBr): v/ $\mathrm{cm}^{-1}$ $=3423-3233\left(2 \mathrm{NH}_{2}, 3 \mathrm{NH}\right), 3053(\mathrm{CH}$ aromatic $), 2960$ $\left(\mathrm{CH}_{3}\right), 2884\left(\mathrm{CH}_{2}\right), 2760(\mathrm{CH}), 2224,2221(2 \mathrm{CN}), 1680$, 1672, $1662(3 \mathrm{CO}), 1659(\mathrm{C}=\mathrm{N}), 1651(\mathrm{C}=\mathrm{C}) .{ }^{1} \mathrm{H}$ NMR $\left(\mathrm{DMSO}_{6}\right) \delta=3.25\left(\mathrm{~s}, 3 \mathrm{H}, \mathrm{OCH}_{3}\right), 3.44\left(\mathrm{~s}, 2 \mathrm{H}, \mathrm{CH}_{2}\right)$, $4.51,4.73\left(2 \mathrm{~s}, 4 \mathrm{H}, \mathrm{D}_{2} \mathrm{O}\right.$-exchangeable, $\left.2 \mathrm{NH}_{2}\right), 5.55(\mathrm{~s}, 1 \mathrm{H}$, imidazolidindione ring), 7.39- 7.61 (d.d, $4 \mathrm{H}, \mathrm{C}_{6} \mathrm{H}_{4}$ ), 8.56, 8.74, $9.22\left(3 \mathrm{~s}, 3 \mathrm{H}, \mathrm{D}_{2} \mathrm{O}\right.$-exchangeable, $\left.3 \mathrm{NH}\right) .{ }^{13} \mathrm{C} \mathrm{NMR}$ : $\delta=41.2\left(\mathrm{CH}_{3}\right), 49.7\left(\mathrm{CH}_{2}\right), 63.9(\mathrm{CH}), 76.7,86.8(\mathrm{C}=\mathrm{C})$, $97.9(\mathrm{C}=\mathrm{N}), 117.2,119.8(2 \mathrm{CN}), 123.9,126.7,129.9$, 132.5, 136.2, 138.9, 140.7 (pyrimidine $\mathrm{C}, \mathrm{C}_{6} \mathrm{H}_{4}$ ), 162.2, 164.8, $167.8(3 \mathrm{C}=\mathrm{O})$. MS (relative intensity) $\mathrm{m} / \mathrm{z}: 479$ $\left(\mathrm{M}^{+}, 23.3 \%\right)$. Calcd for $\mathrm{C}_{20} \mathrm{H}_{17} \mathrm{~N}_{9} \mathrm{O}_{4} \mathrm{~S}$ (479.47): $\mathrm{C}, 50.10$; $\mathrm{H}, 3.57$; N, 26.29; S, 6.69\%. Found: C, 50.34; H, 3.28; N, 26.57; S, $6.41 \%$.
General procedure for the synthesis of compound: 6-amino-2-(4,6-diamino-1-(2,5-dioxoimidazolidin-4-yl)2-oxo-1,2-dihydropyrimidin-5-yl)-4-(4-methoxyphenyl)nicotinonitrile (19)

The solution of compound $17 \mathbf{a}(0.447 \mathrm{~g}, 0.001 \mathrm{~mol})$ in sodium ethoxide $(0.001 \mathrm{~mol})$ [prepared by dissolving sodium metal $(0.023 \mathrm{~g}, 0.001 \mathrm{~mol})$ in absolute ethanol (50 $\mathrm{mL})]$. The reaction was heated under reflux for $4 \mathrm{~h}$ and then evaporated under vacuum. The product was triturated with ethanol and the formed product was collected by filtration.

Compound 19: Yellow crystals from ethanol, yield $57 \%, 0.255$ g, m.p. $207-209^{\circ} \mathrm{C}$. IR (KBr): v $/ \mathrm{cm}^{-1}=3462-$ $3220\left(3 \mathrm{NH}_{2}, 2 \mathrm{NH}\right), 3054(\mathrm{CH}$ aromatic $), 2985\left(\mathrm{CH}_{3}\right)$, $2766(\mathrm{CH}), 2221(\mathrm{CN}), 1688,1672,1664(3 \mathrm{CO}), 1655$ $(\mathrm{C}=\mathrm{N}), 1647(\mathrm{C}=\mathrm{C}) .{ }^{1} \mathrm{H}$ NMR $\left(\mathrm{DMSO}_{-} \mathrm{d}_{6}\right) \delta=3.68(\mathrm{~s}$, $\left.3 \mathrm{H}, \mathrm{OCH}_{3}\right), 4.38,4.93,5.33\left(3 \mathrm{~s}, 6 \mathrm{H}, \mathrm{D}_{2} \mathrm{O}\right.$-exchangeable, $\left.3 \mathrm{NH}_{2}\right), 5.77(\mathrm{~s}, 1 \mathrm{H}$, imidazolidindione ring), $7.14(\mathrm{~s}, 1 \mathrm{H}$, pyridine), 7.28-7.49 (d.d, $\left.4 \mathrm{H}, \mathrm{C}_{6} \mathrm{H}_{4}\right), 8.41,8.82(2 \mathrm{~s}, 2 \mathrm{H}$, $\mathrm{D}_{2} \mathrm{O}$-exchangeable, $\left.2 \mathrm{NH}\right) .{ }^{13} \mathrm{C}$ NMR: $\delta=40.4\left(\mathrm{CH}_{3}\right)$, $63.3(\mathrm{CH}), 117.8(\mathrm{CN}), 120.6,123.9,125.2,127.6,131.1$, 133.5, 136.2, 138.4, 140.7, 142.6, 144.5, 145.8 (Pyridine $\mathrm{C}$, pyrimidine $\left.\mathrm{C}, \mathrm{C}_{6} \mathrm{H}_{4}\right), 160.8,163.3,166.7(3 \mathrm{C}=\mathrm{O})$. MS (relative intensity) $\mathrm{m} / \mathrm{z}: 447\left(\mathrm{M}^{+}, 30.5 \%\right)$. Calcd for $\mathrm{C}_{20} \mathrm{H}_{17} \mathrm{~N}_{9} \mathrm{O}_{4}$ (447.41): C, 53.69; H, 3.83; N, 28.18\%. Found: C, 53.44; H, 4.09; N, 28.37\%.

General procedure for the synthesis of compounds: 2-((4,6-diamino - 1-(2,5-dioxo-imidazolidin-4-yl) -2-oxo -1,2-dihydropyrimidin -5-yl) (imino) methyl) -4-hydrazinyl-3-(4-methoxyphenyl)but-2-enenitrile (21a) and 2-((4,6-diamino-1-(2,5-dioxo-imidazol-idin4-yl)-2-oxo-1,2-dihydropyrimidin-5-yl) (imino) methyl)3-(4-methoxy -phenyl)-4-(2-phenyl- hydrazinyl)but-2enenitrile (21b)

Either hydrazine hydrate (20a) $(0.1 \mathrm{~g}, 0.002 \mathrm{~mol})$ or phenylhydrazine (20b) (0.22 g, 0.002) was added to a solution of compound $15(1.002 \mathrm{~g}, 0.002 \mathrm{~mol})$ in ethanol $(50 \mathrm{~mL})$. The reaction mixture was heated under reflux for $4 \mathrm{~h}$ and then poured onto an ice/water mixture containing a few drops of hydrochloric acid. The formed solid product was collected by filtration.

Compound 21a: Pale yellow crystals from ethanol, yield $74 \%, 0.67$ g, m.p. $221-223{ }^{\circ} \mathrm{C}$. IR (KBr): v/cm ${ }^{-1}$ $=3389-3212\left(3 \mathrm{NH}_{2}, 4 \mathrm{NH}\right), 3050(\mathrm{CH}$ aromatic $), 2974$ $\left(\mathrm{CH}_{3}\right), 2881\left(\mathrm{CH}_{2}\right), 2760(\mathrm{CH}), 2227(\mathrm{CN}), 1683$, 1667, 1660 (3CO), $1655(\mathrm{C}=\mathrm{N}), 1649(\mathrm{C}=\mathrm{C}) .{ }^{1} \mathrm{H}$ NMR $\left(\mathrm{DMSO}_{\mathrm{d}}\right) \delta=3.19\left(\mathrm{~s}, 3 \mathrm{H}, \mathrm{OCH}_{3}\right), 3.28\left(\mathrm{~s}, 2 \mathrm{H}, \mathrm{CH}_{2}\right)$, $4.58,5.12,5.28\left(3 \mathrm{~s}, 6 \mathrm{H}, \mathrm{D}_{2} \mathrm{O}\right.$-exchangeable, $\left.3 \mathrm{NH}_{2}\right), 5.71$ (s, $1 \mathrm{H}$, imidazolidindione ring), 6.83-7.17 (d.d, $4 \mathrm{H}, \mathrm{C}_{6} \mathrm{H}_{4}$ ), 
8.43, 8.68, 8.77, $9.53\left(4 \mathrm{~s}, 4 \mathrm{H}, \mathrm{D}_{2} \mathrm{O}\right.$-exchangeable, $\left.4 \mathrm{NH}\right)$. ${ }^{13} \mathrm{C}$ NMR: $\delta=37.5\left(\mathrm{CH}_{3}\right), 53.3\left(\mathrm{CH}_{2}\right), 66.7(\mathrm{CH}), 79.4$, $86.4(\mathrm{C}=\mathrm{C}), 107.6(\mathrm{C}=\mathrm{N}), 115.7(\mathrm{CN}), 120.5,125.9,128.2$, 132.3, 134.7, 136.7, 138.9 (pyrimidine $\left.\mathrm{C}, \mathrm{C}_{6} \mathrm{H}_{4}\right), 164.4$, 166.9, $170.2(3 \mathrm{C}=\mathrm{O})$. MS (relative intensity) $\mathrm{m} / \mathrm{z}: 452$ $\left(\mathrm{M}^{+}, 27.4 \%\right)$. Calcd for $\mathrm{C}_{19} \mathrm{H}_{20} \mathrm{~N}_{10} \mathrm{O}_{4}(452,43)$ : C, 50.44; H, 4.46; N, 30.96\%. Found: C, 50.71; H, 4.73; N, 30.68\%.

Compound 21b: Pale yellow crystals from ethanol, yield $65 \%, 0.688$ g, m.p. $240-242{ }^{\circ} \mathrm{C}$. IR (KBr): v/ $\mathrm{cm}^{-1}=3368-3188\left(2 \mathrm{NH}_{2}, 5 \mathrm{NH}\right), 3056(\mathrm{CH}$ aromatic $)$, $2988\left(\mathrm{CH}_{3}\right), 2879\left(\mathrm{CH}_{2}\right), 2758(\mathrm{CH}), 2225(\mathrm{CN}), 1688$, 1666, $1661(3 \mathrm{CO}), 1657(\mathrm{C}=\mathrm{N}), 1650(\mathrm{C}=\mathrm{C}) .{ }^{1} \mathrm{H}$ NMR $\left(\mathrm{DMSO}_{6}\right) \delta=3.12\left(\mathrm{~s}, 3 \mathrm{H}, \mathrm{OCH}_{3}\right), 3.22\left(\mathrm{~s}, 2 \mathrm{H}, \mathrm{CH}_{2}\right)$, $5.17,5.44\left(2 \mathrm{~s}, 4 \mathrm{H}, \mathrm{D}_{2} \mathrm{O}\right.$-exchangeable, $\left.2 \mathrm{NH}_{2}\right), 5.55(\mathrm{~s}$, $1 \mathrm{H}$, imidazolidindione ring), 6.91-7.12 (d.d, $4 \mathrm{H}, \mathrm{C}_{6} \mathrm{H}_{4}$ ), 7.38-7.53 (m, 5H, $\left.\mathrm{C}_{6} \mathrm{H}_{5}\right), 8.22,8.51,8.79,9.11,9.58(5 \mathrm{~s}$, $5 \mathrm{H}, \mathrm{D}_{2} \mathrm{O}$-exchangeable, $\left.5 \mathrm{NH}\right) .{ }^{13} \mathrm{C}$ NMR: $\delta=39.4\left(\mathrm{CH}_{3}\right)$, $51.7\left(\mathrm{CH}_{2}\right), 69.5(\mathrm{CH}), 78.8,83.2(\mathrm{C}=\mathrm{C}), 110.4(\mathrm{C}=\mathrm{N})$, $117.6(\mathrm{CN}), 120.3,122.5,124.7,127.1,130.8,132.6$, $134.8,136.5,139.6,141.2,143.4,146.4$ (pyrimidine $\mathrm{C}$, $\left.\mathrm{C}_{6} \mathrm{H}_{4}, \mathrm{C}_{6} \mathrm{H}_{5}\right), 163.3,165.8,167.8(3 \mathrm{C}=\mathrm{O})$. MS (relative intensity) $\mathrm{m} / \mathrm{z}: 528\left(\mathrm{M}^{+}, 23.6 \%\right)$. Calcd for $\mathrm{C}_{25} \mathrm{H}_{24} \mathrm{~N}_{10} \mathrm{O}_{4}$ (528.52): C, 56.81; H, 4.58; N, 26.50\%. Found: C, 56.55; $\mathrm{H}, 4.33 ; \mathrm{N}, 26.21 \%$.

General procedure for the synthesis of compounds: 5-(4,6-diamino-5-((1,2-diamino-4-(4-methoxyphenyl)$1 \mathrm{H}$-pyrrol- 3-yl) (imino)methyl)-2-oxopyrimidin-1(2H)-yl) imidazolidine-2,4-dione (23a) and 5-(4,6-diamino-5((2-amino-4-(4-methoxyphenyl)-1-(phenylamino)- $1 \mathrm{H}$ pyrrol-3-yl)(imino)methyl)-2-oxopyrimidin-1(2H)-yl) imidazolidine-2,4-dione (23b)

The reactions began either with solutions of compound $21 \mathrm{a}(0.452 \mathrm{~g}, 0.001 \mathrm{~mol})$ or compound 21b $(0.528 \mathrm{~g}, 0.001 \mathrm{~mol})$ in sodium ethoxide $(0.001 \mathrm{~mol})$ in absolute ethanol $(50 \mathrm{~mL})$. The reaction was heated under reflux for $3 \mathrm{~h}$ and then evaporated under vacuum. The product was triturated with ethanol and the formed product was collected by filtration.

Compound 23a: Creamy white crystals from ethanol, yield $57 \%, 0.258$ g, m.p. $178-180{ }^{\circ} \mathrm{C}$. IR (KBr): $\mathrm{v} / \mathrm{cm}^{-1}=3411-3264\left(4 \mathrm{NH}_{2}, 3 \mathrm{NH}\right), 3055(\mathrm{CH}$ aromatic $)$, $2981\left(\mathrm{CH}_{3}\right), 2768(\mathrm{CH}), 1689,1668,1663(3 \mathrm{CO}), 1651$ $(\mathrm{C}=\mathrm{N}), 1645(\mathrm{C}=\mathrm{C}) .{ }^{1} \mathrm{H}$ NMR $\left(\mathrm{DMSO}^{-} \mathrm{d}_{6}\right) \delta=3.27(\mathrm{~s}, 3 \mathrm{H}$, $\left.\mathrm{OCH}_{3}\right), 4.38,4.59,4.95,5.23\left(4 \mathrm{~s}, 8 \mathrm{H}, \mathrm{D}_{2} \mathrm{O}\right.$-exchangeable, $\left.4 \mathrm{NH}_{2}\right), 5.63$ (s, $1 \mathrm{H}$, imidazolidindione ring), 6.95-7.28 $\left(\mathrm{m}, 4 \mathrm{H}, \mathrm{C}_{6} \mathrm{H}_{4}, 1 \mathrm{H}\right.$, pyrrole), 8.51, 8.77, $9.38\left(3 \mathrm{~s}, 3 \mathrm{H}, \mathrm{D}_{2} \mathrm{O}-\right.$ exchangeable, $3 \mathrm{NH}) .{ }^{13} \mathrm{C}$ NMR: $\delta=39.2\left(\mathrm{CH}_{3}\right), 64.4(\mathrm{CH})$, $103.8(\mathrm{C}=\mathrm{N}), 121.7,123.6,126.7,128.9,131.4,133.6$,
136.5, 138.5, 140.2 , 143.4, 145.1 (pyrrole $\mathrm{C}$, pyrimidine $\mathrm{C}$, $\left.\mathrm{C}_{6} \mathrm{H}_{4}\right), 167.1,169.8,173.4(3 \mathrm{C}=\mathrm{O})$. MS (relative intensity) $\mathrm{m} / \mathrm{z}: 452\left(\mathrm{M}^{+}, 21.5 \%\right)$. Calcd for $\mathrm{C}_{19} \mathrm{H}_{20} \mathrm{~N}_{10} \mathrm{O}_{4}(452,43)$ : C, 50.44; H, 4.46; N, 30.96\%. Found: C, 50.18; H, 4.69; $\mathrm{N}, 30.72 \%$.

Compound 23b: Pale yellow crystals from ethanol, yield $55 \%, 0.29$ g, m.p. $150-152{ }^{\circ} \mathrm{C}$. IR $(\mathrm{KBr}): \mathrm{v} / \mathrm{cm}^{-1}=$ 3386-3187 $\left(3 \mathrm{NH}_{2}, 4 \mathrm{NH}\right), 3053$ (CH aromatic), 2975 $\left(\mathrm{CH}_{3}\right), 2782(\mathrm{CH}), 1685,1671,1665(3 \mathrm{CO}), 1656(\mathrm{C}=\mathrm{N})$, $1650(\mathrm{C}=\mathrm{C}) .{ }^{1} \mathrm{H}$ NMR $\left(\mathrm{DMSO}_{6}\right) \delta=3.41\left(\mathrm{~s}, 3 \mathrm{H}, \mathrm{OCH}_{3}\right)$, 4.44, 4.67, 5.21 (3s, $6 \mathrm{H}, \mathrm{D}_{2} \mathrm{O}$-exchangeable, $\left.3 \mathrm{NH}_{2}\right), 5.54$ (s, $1 \mathrm{H}$, imidazolidindione ring), 6.86-7.37 $\left(\mathrm{m}, 4 \mathrm{H}, \mathrm{C}_{6} \mathrm{H}_{4}\right.$, $5 \mathrm{H}, \mathrm{C}_{6} \mathrm{H}_{5}, 1 \mathrm{H}$, pyrrole), 8.43, 8.68, 9.17, $9.56(4 \mathrm{~s}, 4 \mathrm{H}$, $\mathrm{D}_{2} \mathrm{O}$-exchangeable, $\left.4 \mathrm{NH}\right) .{ }^{13} \mathrm{C}$ NMR: $\delta=35.7\left(\mathrm{CH}_{3}\right), 57.4$ $(\mathrm{CH}), 101.4(\mathrm{C}=\mathrm{N}), 120.2,121.9,123.3,125.8,127.5$, 130.1, 132.7, 134.6, 136.7, 139.1, 141.5, 144.1, 146.3, 147.5 (pyrrole $\mathrm{C}$, pyrimidine $\mathrm{C}, \mathrm{C}_{6} \mathrm{H}_{4}, \mathrm{C}_{6} \mathrm{H}_{5}$ ), 163.3, 169.4, $171.8(3 \mathrm{C}=\mathrm{O})$. MS (relative intensity) $\mathrm{m} / \mathrm{z}: 528$ $\left(\mathrm{M}^{+}, 28.3 \%\right)$. Calcd for $\mathrm{C}_{25} \mathrm{H}_{24} \mathrm{~N}_{10} \mathrm{O}_{4}$ (528.52): C, 56.81; H, 4.58; N, 26.50\%. Found: C, 56.55; H, 4.86; N, 26.33\%.

General procedure for the synthesis of compounds: 5-(4-amino-6-imino-2-oxo-5-(1-phenylethylidene)-5,6dihydropyrimidin-1(2H)-yl)imidazolidine-2,4-diones (25a), 5-(4-amino-6-imino-2-oxo-5-(2-phenylhydrazono5,6-dihydropyrimidin-1(2H)-yl) imidazol- idine-2,4dione (25b) and 5-(4-amino-5-(2-(4-chlorophenyl) hydrazono)-6-imino-2-oxo-5,6-dihydro-pyrimidin-1(2H)yl)imidazolidine-2,4-dione (25c)

Either compound 24a (0.505 g, $0.003 \mathrm{~mol}), \mathbf{2 4 b}$ $(0.614 \mathrm{~g}, 0.003 \mathrm{~mol})$ or $\mathbf{2 4 c}(0.474 \mathrm{~g}, 0.003 \mathrm{~mol})$ was added to a solution of 5-ureidohydantion (2) $(0.474 \mathrm{~g}, 0.003 \mathrm{~mol})$ in $50 \mathrm{~mL}$ of ethanol containing dimethylformamide $(5.0 \mathrm{~mL})$ and triethylamine $(1.0 \mathrm{~mL})$ as a catalyst. The reaction mixture was heated under reflux for $5 \mathrm{~h}$, cooled and poured onto ice containing a few drops of $\mathrm{HCl}$. The formed solid product was filtered out.

Compound 25a: Pale brown crystals from ethanol, yield $61 \%, 0.597$ g, m.p. $251-253{ }^{\circ} \mathrm{C}$. IR (KBr): $\mathrm{v} / \mathrm{cm}^{-1}=3407-3326\left(\mathrm{NH}_{2}, 3 \mathrm{NH}\right), 3051(\mathrm{CH}$ aromatic $)$, $2978\left(\mathrm{CH}_{3}\right), 2734(\mathrm{CH}), 1688,1671,1662(3 \mathrm{CO}), 1657$ $(\mathrm{C}=\mathrm{N}), 1647(\mathrm{C}=\mathrm{C}) .{ }^{1} \mathrm{HNMR}(\mathrm{DMSO}) \delta=1.87(\mathrm{~s}, 3 \mathrm{H}$, $\left.\mathrm{CH}_{3}\right), 4.63\left(\mathrm{~s}, 2 \mathrm{H}, \mathrm{D}_{2} \mathrm{O}\right.$-exchangeable, $\left.\mathrm{NH}_{2}\right), 5.65(\mathrm{~s}, 1 \mathrm{H}$, imidazolidindione ring), 7.27-7.44 (m, $\left.5 \mathrm{H}, \mathrm{C}_{6} \mathrm{H}_{5}\right), 8.22$, 8.46, 9.37 (3s, 3H, $\mathrm{D}_{2} \mathrm{O}$-exchangeable, $\left.3 \mathrm{NH}\right) .{ }^{13} \mathrm{C}$ NMR: $\delta=23.3\left(\mathrm{CH}_{3}\right), 61.4(\mathrm{CH}), 86.4(\mathrm{C}=\mathrm{C}), 118.7,123.5$, 126.7, 128.9, 130.2, 133.6, 137.4, 139.3 (pyrimidine $\mathrm{C}$, $\left.\mathrm{C}_{6} \mathrm{H}_{5}\right), 160.2,162.7,165.6(3 \mathrm{C}=\mathrm{O})$. MS (relative intensity) $\mathrm{m} / \mathrm{z}: 326\left(\mathrm{M}^{+}, 19.8 \%\right)$. Calcd for $\mathrm{C}_{15} \mathrm{H}_{14} \mathrm{~N}_{6} \mathrm{O}_{3}(326.31)$ : $\mathrm{C}$, 
55.21; H, 4.32; N, 25.75\%. Found: C, 55.48; H, 4.05; N, $25.49 \%$.

Compound 25b: Brown crystals from ethanol, yield 53\%, 0.522 g, m.p. $197-199{ }^{\circ} \mathrm{C}$. IR (KBr): $\mathrm{v} / \mathrm{cm}^{-1}=3428-3335\left(\mathrm{NH}_{2}, 4 \mathrm{NH}\right), 3053$ (CH aromatic), 2766 $(\mathrm{CH}), 1684,1672,1664(3 \mathrm{CO}), 1656(\mathrm{C}=\mathrm{N}), 1649(\mathrm{C}=\mathrm{C})$. ${ }^{1} \mathrm{HNMR}(\mathrm{DMSO}) \delta=4.55$ (s, $2 \mathrm{H}, \mathrm{D}_{2} \mathrm{O}$-exchangeable, $\mathrm{NH}_{2}$ ), $5.43(\mathrm{~s}, 1 \mathrm{H}$, imidazolidindione ring), 7.18-7.37 (m, 5H, $\left.\mathrm{C}_{6} \mathrm{H}_{5}\right), 8.33,8.49,8.74,9.37\left(4 \mathrm{~s}, 4 \mathrm{H}, \mathrm{D}_{2} \mathrm{O}\right.$-exchangeable, $4 \mathrm{NH}) .{ }^{13} \mathrm{C} \mathrm{NMR:} \delta=65.5(\mathrm{CH}), 120.4,122.7,125.9,127.4$, 130.4, 132.8, 135.8, 138.7 (pyrimidine $\mathrm{C}, \mathrm{C}_{6} \mathrm{H}_{5}$ ), 161.7, $163.9,165.5(3 \mathrm{C}=\mathrm{O})$. MS (relative intensity) $\mathrm{m} / \mathrm{z}: 328\left(\mathrm{M}^{+}\right.$, 15.7\%). Calcd for $\mathrm{C}_{13} \mathrm{H}_{12} \mathrm{~N}_{8} \mathrm{O}_{3}$ (328.29): C, 47.56; H, 3.68; N, 34.13\%. Found: C, 47.31; H, 3.94; N, 34.37\%.

Compound 25c: Brown crystals from ethanol, yield $57 \%, 0.62$ g, m.p. $218-220^{\circ} \mathrm{C}$. IR (KBr): v $/ \mathrm{cm}^{-1}=3444-$ $3352\left(\mathrm{NH}_{2}, 4 \mathrm{NH}\right), 3057(\mathrm{CH}$ aromatic), $2761(\mathrm{CH}), 1682$, 1670, $1663(3 \mathrm{CO}), 1653(\mathrm{C}=\mathrm{N}), 1647(\mathrm{C}=\mathrm{C}) .{ }^{1} \mathrm{HNMR}$ (DMSO) $\delta=4.72\left(\mathrm{~s}, 2 \mathrm{H}, \mathrm{D}_{2} \mathrm{O}\right.$-exchangeable, $\left.\mathrm{NH}_{2}\right), 5.66$ (s, $1 \mathrm{H}$, imidazolidindione ring), 7.39-7.54 (d.d, $4 \mathrm{H}, \mathrm{C}_{6} \mathrm{H}_{4}$ ), $8.38,8.62,8.88,9.28\left(4 \mathrm{~s}, 4 \mathrm{H}, \mathrm{D}_{2} \mathrm{O}\right.$-exchangeable, $\left.4 \mathrm{NH}\right)$. ${ }^{13} \mathrm{C}$ NMR: $\delta=54.8(\mathrm{CH}), 121.4,123.6,125.7,128.5$, $131.5,133.9,135.7,139.5$ (pyrimidine $\left.\mathrm{C}, \mathrm{C}_{6} \mathrm{H}_{4}\right), 162.8$, 164.7, $167.3(3 \mathrm{C}=\mathrm{O})$. MS (relative intensity) $\mathrm{m} / \mathrm{z}: 362$ $\left(\mathrm{M}^{+}, 15.7 \%\right)$. Calcd for $\mathrm{C}_{13} \mathrm{H}_{11} \mathrm{ClN}_{8} \mathrm{O}_{3}(362.73)$ : C, 43.05; H, 3.06; N, 30.89\%. Found: C, 43.33; H, 3.34; N, 30.62\%.

\section{Pharmacology}

\section{Analgesic activity}

Analgesic activity was introduced by the tail flick method (Fadeyi et al., 2004; Vogel, 2002). Healthy albino mice weighing $20.0 \mathrm{~g}$ to $30.0 \mathrm{~g}$ were divided into different groups with six animals in each group. The control group received a $0.5 \% \mathrm{w} / \mathrm{v}$ carboxymethylcellulose (CMC) solution and the treated groups were given a $132 \mu \mathrm{mol} / \mathrm{kg}$ orally dose of compounds $3,7,9 \mathbf{a}, \mathbf{b}, 11,13 \mathbf{a}, \mathbf{b}, 15,17 \mathbf{a}$, b, 19, 21a, b, 23a, b and 25a, b, c. The reaction times were noted at $2 \mathrm{~h}$ and $4 \mathrm{~h}$ intervals after drug administration. The percentage analgesic activity was calculated by the following formula:-

\section{Percentage analgesic activity $=\mathrm{T}_{2}-\mathrm{T}_{1} / \mathrm{T}_{1} \times 100$}

where:- $T_{1}$ is the normal reaction time; $T_{2}$ is the reaction time after treatment.

\section{Antipyretic activity}

Healthy Wistar rats were given s.c. $10 \mathrm{~mL} / \mathrm{kg}$ of a $20 \%$ aqueous suspension of sterilized brewer's yeast powder (Fadeyi et al., 2004; Vogel, 2002) weighting between $150 \mathrm{~g}$ and $200 \mathrm{~g}$. Eighteen hours later, the animals showing an increase in rectal temperature greater than 0.5 ${ }^{\circ} \mathrm{C}$ were selected. The control group received a $0.5 \% \mathrm{w} / \mathrm{v}$ carboxymethylcellulose solution and the treated groups received a of $132 \mu \mathrm{mol} / \mathrm{kg}$ dose of compounds $\mathbf{3}, \mathbf{7}, \mathbf{9 a}$, b, 11, 13a, b, 15, 17a, b, 19, 21a, b, 23a, b and 25a, b, c. Rectal temperatures were noted using digital thermometer 30 minute before (pretreated) and at $1 \mathrm{~h}, 2 \mathrm{~h}$ and $4 \mathrm{~h}$ after administration of the dose.

\section{Anti-inflammatory activity}

The anti-inflammatory activity was examined using a hind paw edema method on albino rats of either six (Fadeyi et al., 2004; Vogel, 2002). A freshly prepared of carrageenan solution $(0.1 \mathrm{~mL}, 1 \% \mathrm{w} / \mathrm{v})$ was injected into the sub-plantar surface of the right hind limb of each animal. The control group received a $0.5 \% \mathrm{w} / \mathrm{v}$ CMC solution and the treated groups were orally given a $132 \mu \mathrm{mol} / \mathrm{kg}$ dose of compounds $3,7,9 \mathbf{a}, \mathbf{b}, 11,13 \mathbf{a}, \mathbf{b}, 15,17 \mathbf{a}, \mathbf{b}, 19,21 \mathbf{a}$, $\mathbf{b}, \mathbf{2 3 a}, \mathbf{b}$ and 25a, b, c 30 minute before carrageenan. The volume of each paw was measured with a plethysmometer at $2 \mathrm{~h}$ and $4 \mathrm{~h}$ intervals after carrageenan injection. The percentage inhibition of edema was calculated by the following formula:

\section{Percentage inhibition of edema: $\mathrm{V}_{\mathrm{C}}-\mathrm{V}_{\mathrm{T}} / \mathrm{V}_{\mathrm{C}} \times 100$}

where: $\mathrm{V}_{\mathrm{C}}$ is the paw volume of control animal; $\mathrm{V}_{\mathrm{T}}$ is the paw volume of treated animals (standard/test compound).

\section{RESULTS AND DISCUSSION}

This study was a continuation of our efforts aimed at the synthesis of new heterocyclic compounds with significant biological potential (El-Sharkawy et al., 2012; Mohareb, El-Sharkawy, Sherif, 2008). The goals of this work were to study the possibility of using compounds $\mathbf{2}$ and $\mathbf{3}$ in heterocyclic synthesis to produce the pyridopyrimidine derivative 7 ; thiophene derivatives $\mathbf{9 a}, \mathbf{b}$; coumarin derivative 11; pyrimidine derivatives $\mathbf{1 3}, \mathbf{1 5}, \mathbf{1 7} \mathbf{a}, \mathbf{b}, \mathbf{2 1} \mathbf{a}, \mathbf{b}$; pyridine derivative 19; pyrazole derivatives 23a,b and iminopyrimidine derivatives $\mathbf{2 5 a}, \mathbf{b}, \mathbf{c}$, as well as biologically evaluate these compounds for analgesic, antipyretic and anti-inflammatory activities. The reaction of $\beta$-amino- $\alpha, \gamma$-dicyanocrotono- nitrile (1) with 5-ureidohydantion (2) using triethylamine as catalyst produced compound $\mathbf{3}$. The latter product underwent cyclization in the presence of piperidine. Four isomeric 


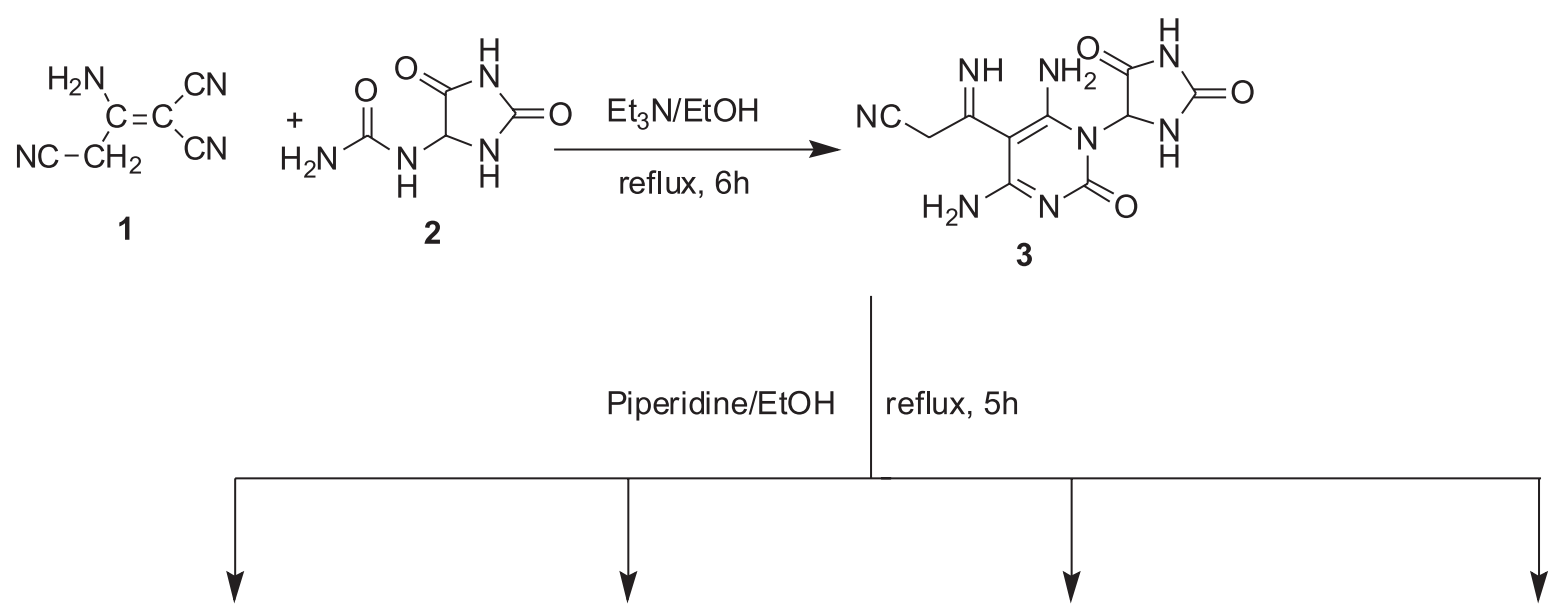<smiles>N=c1c2c(N)cc(N)nc2nc(O)n1C1NC(=O)NC1=O</smiles>

6

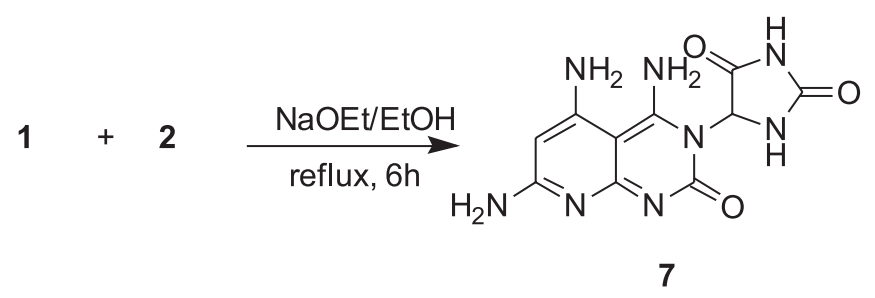

FIGURE 1 - Synthesis rout for compounds 3 and 7.

structures were considered, including 4,5,6 and 7. The ${ }^{1} \mathrm{HNMR}$ spectral data showed that the final product contained three singlets at $\delta=4.78,4.93,5.27 \mathrm{ppm}$ and two singlets at $\delta=8.73,9.95 \mathrm{ppm}$ which represented the presence of $3 \mathrm{NH}_{2}$ and $2 \mathrm{NH}$ groups, respectively; thus, the structures of compounds $\mathbf{4 , 5}$ and $\mathbf{6}$ were ruled out, as those latter structures only containing $2 \mathrm{NH}_{2}$ groups. Additionally, structure 6 contained an $\mathrm{OH}$ group which it was absent in the analytical and spectral data. In contrast, compound 7 was produced by another pathway, through the reaction of $\beta$-amino- $\alpha, \gamma$-dicyanocrotononitrile (1) with 5-ureidohydantion (2) in the presence of sodium ethoxide directly. Compound 3 reacted with either cyclopentanone (8a) or cyclohexanone (8b) in the presence of elemental sulfur and trimethylamine afforded compounds $\mathbf{9 a}, \mathbf{b}$ respectively. The structures of compounds $\mathbf{9 a}, \mathbf{b}$ were verified by elemental analysis and spectral data. In compound 9a, the ${ }^{1} \mathrm{HNMR}$ spectrum indicated the presence of a multiplet at $\delta=2.07-2.18 \mathrm{ppm}$ which could be assigned to the $3 \mathrm{CH}_{2}$ groups; three singlets at $\delta=4.54$, $4.65,5.81 \mathrm{ppm}$, which indicate the presence of $3 \mathrm{NH}_{2}$ groups; a singlet at $\delta=5.98 \mathrm{ppm}$, which indicate the presence of $1 \mathrm{H}$ of an imidazolidindione ring and three singlets at $\delta=8.52,8.77,9.12 \mathrm{ppm}$ corresponding to $3 \mathrm{NH}$ groups. Coumarin derivative $\mathbf{1 1}$ was formed via the reaction of compound 3 with salicylaldehyde (10) and the structure of the compound was confirmed. The ${ }^{1} \mathrm{HNMR}$ spectrum indicated the presence of two singlets at $\delta=4.76$, $5.25 \mathrm{ppm}$, which indicate the presence of $2 \mathrm{NH}_{2}$ groups; a singlet at $\delta=5.53 \mathrm{ppm}$, which indicates the presence of an $1 \mathrm{H}$ of imidazolidindione ring; a singlet at $\delta=6.73 \mathrm{ppm}$, which indicate the presence of a coumarin $1 \mathrm{H}$; a multiplet at $\delta=7.51-7.66 \mathrm{ppm}$ corresponding to $4 \mathrm{H}$ of benzene ring; and three singlets at $\delta=8.88,9.28,9.55 \mathrm{ppm}$, which indicate the presence of $3 \mathrm{NH}$ groups. Compound $\mathbf{3}$ was also reacted with aryldiazonium salts $\mathbf{1 2 a}, \mathbf{b}$ to afford arylhydrazono derivatives $\mathbf{1 3 a}, \mathbf{b}$ respectively. The elucidation of the structure for these compounds was then 

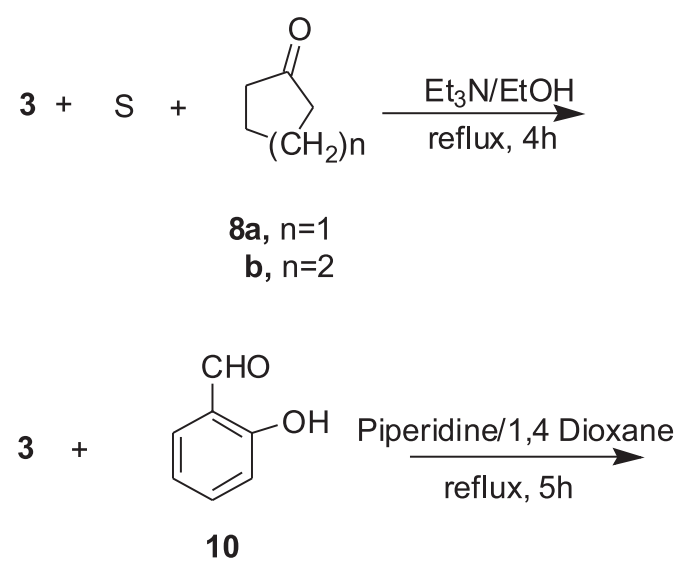

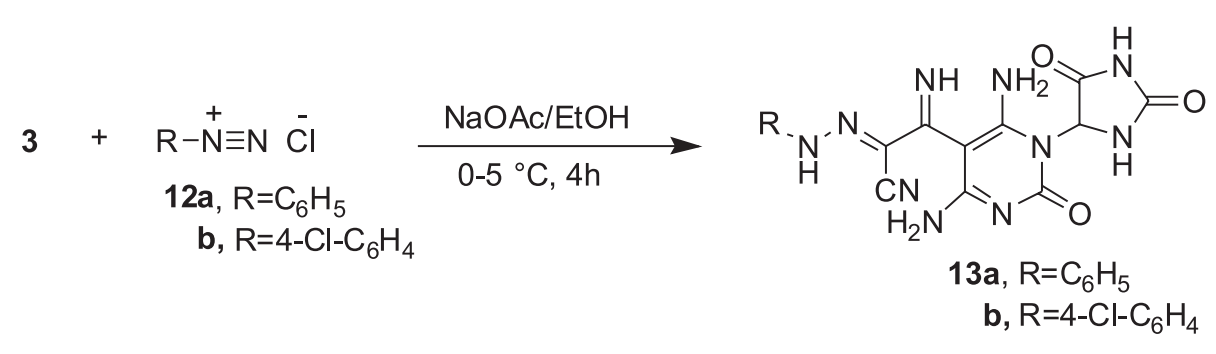<smiles>COc1ccc(C(=O)OCc2ccc(C(C)=C(C)C(=N)c3c(N)nc(=O)n(C4NC(=O)NC4=O)c3N)cc2)cc1</smiles>

FIGURE 2 - Synthesis rout for compounds 9a,b, 11, 13a,b and 15. confirmed. The ${ }^{1} \mathrm{HNMR}$ spectrum for compound 13a showed the presence of two singlets at $\delta=4.54,5.17 \mathrm{ppm}$, which indicate the presence of $2 \mathrm{NH}_{2}$ groups; a singlet at $\delta=5.61 \mathrm{ppm}$, which indicates the presence of $1 \mathrm{H}$ of an imidazolidindione ring; a multiplet at $\delta=7.31-7.62 \mathrm{ppm}$ corresponding to $5 \mathrm{H}$ of benzene ring; and four singlets at $\delta=8.76,9.13,9.38,959 \mathrm{ppm}$ which indicate the presence of $4 \mathrm{NH}$ groups.

The last reaction of compound $\mathbf{3}$, was performed with $\omega$-bromo-4-methoxyacetophenone (14), and the 4-methoxyphenylbutenyl derivative 15 was afforded. The elucidation of this structure was based on analytical and spectral data. Compound $\mathbf{1 5}$ was reacted with either potassium cyanide (16a) or potassium thiocyanate $(\mathbf{1 6} \mathbf{b})$ to form either the 4-methoxyphenylbutenyl cyanide derivative $\mathbf{1 7}$ a or 4-methoxyphenylbutenyl thiocyanide derivative $\mathbf{1 7} \mathbf{b}$, respectively. The structures of compounds $\mathbf{1 7 a}, \mathbf{b}$ were verified by analytical and spectral data. Compound 17a underwent a cyclization in presence of sodium ethoxide to afford pyridine derivative 19 via<smiles>N=C(c1c(N)sc2c1CCC2)c1c(N)nc(=O)n(C2NC(=O)NC2=O)c1N</smiles><smiles>N=C(c1c(N)nc(=O)n(C2NC(=O)NC2=O)c1N)c1cc2ccccc2oc1=O</smiles>

11 formation of intermediate $\mathbf{1 8}$. The structure of compound 19 was then confirmed. The ${ }^{1}$ HNMR spectrum of compound 19 detected the presence of singlet at $\delta=3.68$ ppm, which indicates the presence of $3 \mathrm{H}$ of $\mathrm{CH}_{3}$ group; three singlets at $\delta=4.38,4.935 .33 \mathrm{ppm}$, which indicate the presence of $3 \mathrm{NH}_{2}$ groups; a singlet at $\delta=5.77 \mathrm{ppm}$, which indicates the presence of $1 \mathrm{H}$ of imidazolidindione ring; a singlet at $\delta=7.14 \mathrm{ppm}$ which indicates the presence of $1 \mathrm{H}$ of pyridine ring; a doublet of doublets at $\delta=7.28-7.49$ ppm corresponding to $4 \mathrm{H}$ of benzene ring and two singlets at $\delta=8.41,8.82 \mathrm{ppm}$, which indicate the presence of $2 \mathrm{NH}$ groups. Compound 15 was reacted with either hydrazine hydrate (20a) or phenyl hydrazine (20b) to produce hydrazono derivatives $\mathbf{2 1 a}, \mathbf{b}$, respectively. The structures of these compounds were confirmed by analytical and spectral data. The latter products underwent a cyclization to form pyrrole derivatives 23a,b through the intermediate formation of 22a, $\mathbf{b}$, respectively. The structures of compounds $\mathbf{2 3 a} \mathbf{a}, \mathbf{b}$ were confirmed using analytical and spectral data. The ${ }^{1} \mathrm{HNMR}$ spectrum of compound 23a 


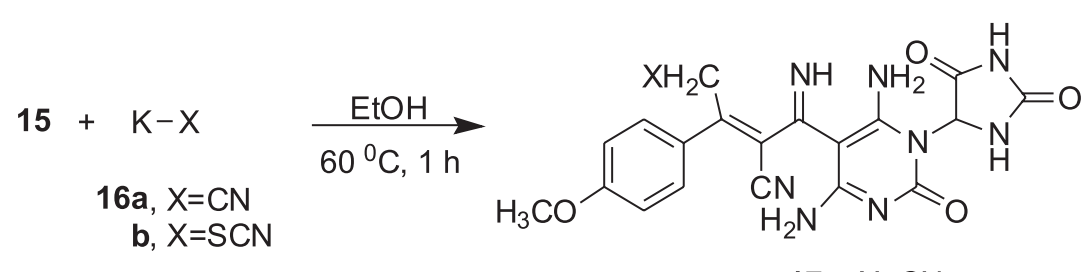

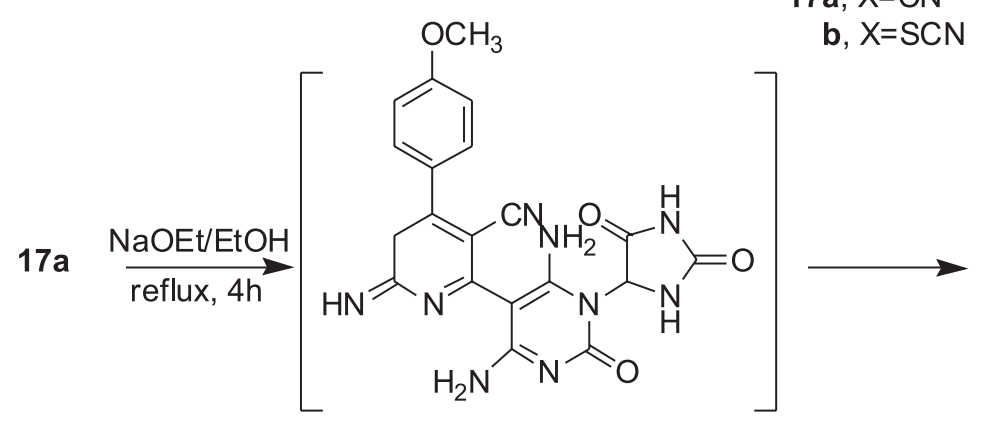

18<smiles>COc1ccc(-c2cc(N)nc(-c3c(N)nc(=O)n(C4NC(=O)NC4=O)c3N)c2C)cc1</smiles>

19<smiles>[R9]NNc1c(C(C)=C(C)c2ccc(OC)cc2)c(N)nc(=O)n1C1NC(=O)NC1=O</smiles>

b, $\mathrm{R}=\mathrm{Ph}$

$$
\begin{aligned}
\text { 21a, } R=H \\
\text { b. } R=P h
\end{aligned}
$$

FIGURE 3 - Synthesis rout for compounds 17a,b, 19 and 21a,b.

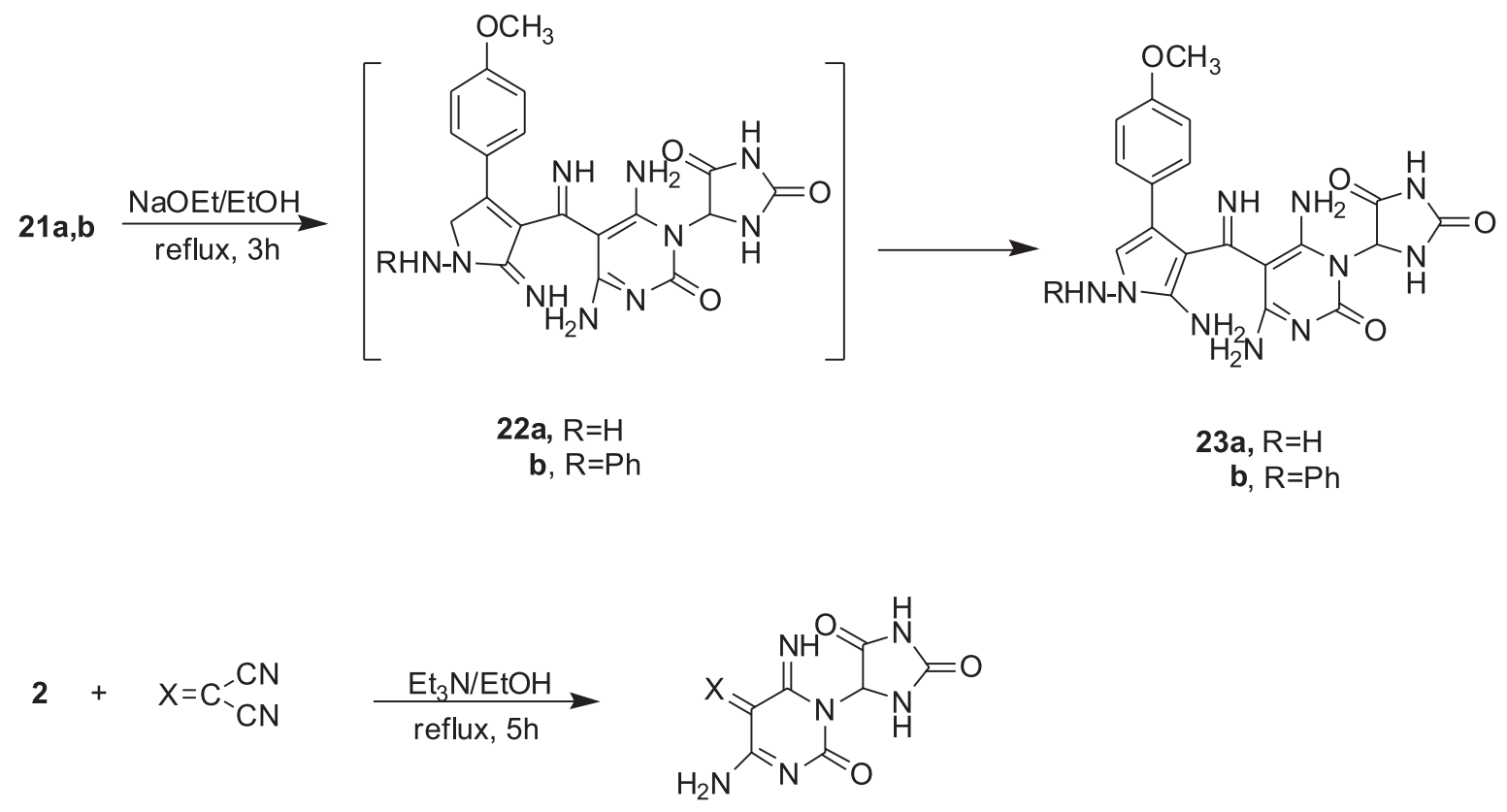

$$
\begin{gathered}
\text { 24a, } \mathrm{X}=\mathrm{Ph}\left(\mathrm{CH}_{3}\right) \mathrm{C} \\
\text { b, } \mathrm{X}=\mathrm{PhNHN} \\
\text { c, } \mathrm{X}=4-\mathrm{ClC}_{6} \mathrm{H}_{4} \mathrm{NHN}
\end{gathered}
$$

25a, $X=P h\left(\mathrm{CH}_{3}\right) \mathrm{C}$

b, $\mathrm{X}=\mathrm{PhNHN}$

c, $\mathrm{X}=4-\mathrm{ClC}_{6} \mathrm{H}_{4} \mathrm{NHN}$

FIGURE 4 - Synthesis rout for compounds 23a,b and 25a,b,c. 
detected the presence of a singlet at $\delta=3.27 \mathrm{ppm}$, which indicates the presence of $3 \mathrm{H}$ from a $\mathrm{CH}_{3}$ group; four singlets at $\delta=4.38,4.59,4.95,5.23 \mathrm{ppm}$, which indicate the presence of $4 \mathrm{NH}_{2}$ groups; a singlet at $\delta=5.63 \mathrm{ppm}$, which indicates the presence of $1 \mathrm{H}$ of imidazolidindione ring; a multiplet at $\delta=6.95-7.28 \mathrm{ppm}$ corresponding to $4 \mathrm{H}$ of benzene ring and $1 \mathrm{H}$ of pyrrole ring and three singlets at $\delta=8.51,8.77,9.38 \mathrm{ppm}$, which indicate the presence of $3 \mathrm{NH}$ groups. Finally 5-ureidohydantion (2) was reacted with compounds $\mathbf{2 4 a}, \mathbf{b}, \mathbf{c}$ to produce iminopyrimidine derivatives $\mathbf{2 5 a}, \mathbf{b}, \mathbf{c}$, respectively and the structure of these compounds were confirmed by analytical and spectral data.

All the synthesized compounds were evaluated for their in vitro analgesic, antipyretic and anti-inflammatory activities. Acetaminophen was used as a reference standard drug. Based on the results from (Tables I and II), it is clear that compounds $\mathbf{1 1}$ and $\mathbf{1 9}$ showed promising actions as analgesic and antipyretic agents. This may be due their containing a coumarin moiety and 4-methoxyphenylpyridine moiety, respectively. In contrast, compounds 7, 9a, b, 13a, b showed moderate analgesic and antipyretic effects. The remaining compounds $\mathbf{3}$, 15, 17a, b, 21a, b, 23a, b, 25a, b, c exhibited poor analgesic and antipyretic effects. Compound $\mathbf{1 1}$ exhibited high significance as an anti-inflammatory agent, which may be due to the presence of the coumarin moiety. Compounds 7, 9b, 13b, 19 were considered as moderate anti-inflammatory effects. The remaining compounds $\mathbf{3}$, 9a, 13a, 15, 17a, b, 21a, b, 23a, b, 25a, b, c exhibited poor biological significance as anti-inflammatory agents (Table III).

TABLE I - Analgesic activities of the synthesized compounds

\begin{tabular}{|c|c|c|c|c|c|}
\hline \multirow{2}{*}{ Comp. No } & \multirow{2}{*}{$\begin{array}{l}\text { Normal reaction } \\
\text { time (sec) }\end{array}$} & \multicolumn{2}{|c|}{ Change in reaction time $(\mathrm{sec}) \pm \mathrm{SEM}$} & \multicolumn{2}{|c|}{$\%$ Analgesic activity \pm SD } \\
\hline & & $2 \mathrm{~h}$ & $4 \mathrm{~h}$ & $2 \mathrm{~h}$ & $4 \mathrm{~h}$ \\
\hline Control & $2.80 \pm 0.15$ & $0.20 \pm 0.014$ & $0.25 \pm 0.018$ & $7.09 \pm 1.45$ & $9.05 \pm 0.87$ \\
\hline 3 & $2.20 \pm 0.09$ & $3.21 \pm 0.15$ & $2.25 \pm 0.08$ & $113.1 \pm 1.57$ & $89.76 \pm 2.23$ \\
\hline 7 & $2.35 \pm 0.08$ & $3.24 \pm 0.10$ & $2.35 \pm 0.07$ & $115.4 \pm 1.38$ & $94.53 \pm 0.44 * *$ \\
\hline $9 a$ & $2.60 \pm 0.07$ & $3.20 \pm 0.08$ & $2.33 \pm 0.06$ & $129.1 \pm 1.65$ & $95.24 \pm 1.53^{* *}$ \\
\hline 9b & $2.57 \pm 0.07$ & $3.17 \pm 0.09$ & $2.36 \pm 0.05$ & $131.3 \pm 1.58$ & $92.56 \pm 1.38 * *$ \\
\hline 11 & $2.38 \pm 0.08$ & $3.18 \pm 0.08$ & $2.43 \pm 0.03$ & $125.1 \pm 1.68$ & $97.56 \pm 0.55 * * *$ \\
\hline $13 \mathbf{a}$ & $2.56 \pm 0.06$ & $3.22 \pm 0.11$ & $2.38 \pm 0.04$ & $133.6 \pm 1.76$ & $95.84 \pm 2.05^{* *}$ \\
\hline $13 b$ & $2.48 \pm 0.06$ & $3.21 \pm 0.08$ & $2.29 \pm 0.07$ & $136.4 \pm 1.48$ & $94.11 \pm 0.43 * *$ \\
\hline 15 & $2.53 \pm 0.08$ & $3.32 \pm 0.15$ & $2.42 \pm 0.08$ & $140.5 \pm 1.54$ & $84.82 \pm 1.23$ \\
\hline $17 \mathbf{a}$ & $2.16 \pm 0.07$ & $3.33 \pm 0.19$ & $1.96 \pm 0.05$ & $145.6 \pm 1.64$ & $86.52 \pm 1.18$ \\
\hline $17 b$ & $2.36 \pm 0.12$ & $3.48 \pm 0.12$ & $2.21 \pm 0.03$ & $138.8 \pm 1.98$ & $89.33 \pm 2.66$ \\
\hline 19 & $2.20 \pm 0.11$ & $3.15 \pm 0.07$ & $2.40 \pm 0.06$ & $119.5 \pm 1.45$ & $96.5 \pm 0.25 * * *$ \\
\hline 21a & $2.25 \pm 0.09$ & $3.44 \pm 0.13$ & $2.25 \pm 0.08$ & $147.1 \pm 1.77$ & $90.5 \pm 1.46$ \\
\hline $21 b$ & $3.15 \pm 0.06$ & $3.38 \pm 0.14$ & $2.05 \pm 0.07$ & $144.3 \pm 1.48$ & $103.55 \pm 1.53$ \\
\hline $23 a$ & $2.60 \pm 0.12$ & $3.47 \pm 0.18$ & $1.98 \pm 0.07$ & $141.1 \pm 1.88$ & $92.4 \pm 2.36^{*}$ \\
\hline $23 b$ & $2.50 \pm 0.12$ & $3.31 \pm 0.13$ & $1.96 \pm 0.06$ & $112.3 \pm 1.93$ & $90.9 \pm 0.95^{*}$ \\
\hline $25 a$ & $2.75 \pm 0.09$ & $3.34 \pm 0.16$ & $2.15 \pm 0.08$ & $117.3 \pm 1.73$ & $89.5 \pm 1.65$ \\
\hline $25 b$ & $2.80 \pm 0.08$ & $3.38 \pm 0.17$ & $2.22 \pm 0.05$ & $113.1 \pm 2.00$ & $104.2 \pm 2.44$ \\
\hline $25 \mathrm{c}$ & $2.65 \pm 0.06$ & $3.39 \pm 0.15$ & $2.18 \pm 0.06$ & $108.3 \pm 1.43$ & $88.7 \pm 2.98$ \\
\hline $\begin{array}{l}\text { Ref. Standard } \\
\text { (Acetaminophen) }\end{array}$ & $2.50 \pm 0.10$ & $3.10 \pm 0.05$ & $2.5 \pm 0.03$ & $128.6 \pm 1.75$ & $103.6 \pm 1.58 * * *$ \\
\hline
\end{tabular}

Note: The reaction time value is the mean \pm SEM $(n=6)$. Statistical analysis was performed with the student's unpaired t-test (Kulkarni, 2003). ${ }^{*} \mathrm{p}<0.05,{ }^{* *} \mathrm{p}<0.01$ and $* * * \mathrm{p}<0.001,132 \mu \mathrm{mol} / \mathrm{kg}$ dose. 
TABLE II - Antipyretic activities of the synthesized compounds

\begin{tabular}{|c|c|c|c|c|c|}
\hline \multirow{2}{*}{ Comp. No } & \multicolumn{2}{|c|}{ Before drug $\left({ }^{\circ} \mathrm{C}\right)$} & \multicolumn{3}{|c|}{ After drug $\left({ }^{\circ} \mathbf{C}\right)$} \\
\hline & $-18 h$ & $0.0 \mathrm{~h}$ & $1 \mathrm{~h}$ & $2 \mathrm{~h}$ & $4 \mathrm{~h}$ \\
\hline Control & $37.47 \pm 5.68$ & $38.22 \pm 0.05$ & $38.08 \pm 0.08$ & $38.04 \pm 0.05$ & $37.83 \pm 0.5$ \\
\hline 3 & $37.35 \pm 0.05$ & $38.17 \pm 0.75$ & $37.88 \pm 0.07$ & $37.58 \pm 0.08$ & $37.33 \pm 0.05$ \\
\hline 7 & $37.41 \pm 0.05$ & $38.38 \pm 0.05$ & $38.12 \pm 0.05$ & $37.55 \pm 0.06$ & $37.28 \pm 0.05^{* *}$ \\
\hline 9a & $37.36 \pm 0.04$ & $38.33 \pm 0.06$ & $37.95 \pm 0.08$ & $37.65 \pm 0.07$ & $37.32 \pm 0.05 * *$ \\
\hline $9 \mathrm{~b}$ & $37.32 \pm 0.05$ & $38.35 \pm 0.06$ & $38.14 \pm 0.07$ & $37.54 \pm 0.08$ & $37.26 \pm 0.05^{* *}$ \\
\hline 11 & $37.23 \pm 0.09$ & $38.40 \pm 0.08$ & $37.93 \pm 0.06$ & $37.63 \pm 0.05$ & $37.44 \pm 0.06 * * *$ \\
\hline $13 a$ & $37.40 \pm 0.05$ & $38.37 \pm 0.05$ & $38.25 \pm 0.08$ & $37.55 \pm 0.06$ & $37.31 \pm 0.05^{* *}$ \\
\hline $13 b$ & $37.44 \pm 0.06$ & $38.39 \pm 0.05$ & $37.98 \pm 0.06$ & $37.38 \pm 0.08$ & $37.27 \pm 0.05^{* *}$ \\
\hline 15 & $37.36 \pm 0.06$ & $38.34 \pm 0.05$ & $38.08 \pm 0.07$ & $37.88 \pm 0.06$ & $37.45 \pm 0.05$ \\
\hline $17 \mathbf{a}$ & $37.34 \pm 0.06$ & $38.37 \pm 0.08$ & $38.05 \pm 0.08$ & $37.68 \pm 0.07$ & $37.39 \pm 0.03$ \\
\hline $17 b$ & $37.44 \pm 0.05$ & $38.39 \pm 0.08$ & $38.18 \pm 0.06$ & $37.91 \pm 0.05$ & $37.58 \pm 0.07$ \\
\hline 19 & $37.28 \pm 0.04$ & $38.31 \pm 0.07$ & $\mathbf{3 8 . 0 5} \pm \mathbf{0 . 0 7}$ & $37.60 \pm 0.05$ & $37.37 \pm 0.04 * * *$ \\
\hline $21 \mathrm{a}$ & $37.30 \pm 0.06$ & $38.27 \pm 0.35$ & $37.95 \pm 0.08$ & $37.58 \pm 0.07$ & $37.31 \pm 0.05$ \\
\hline $21 b$ & $37.43 \pm 0.08$ & $38.41 \pm 0.45$ & $38.12 \pm 0.07$ & $37.74 \pm 0.08$ & $37.47 \pm 0.02$ \\
\hline $23 \mathbf{a}$ & $37.39 \pm 0.08$ & $38.44 \pm 0.53$ & $38.19 \pm 0.08$ & $37.87 \pm 0.05$ & $37.55 \pm 0.04^{*}$ \\
\hline $23 b$ & $37.31 \pm 0.07$ & $38.39 \pm 0.06$ & $38.10 \pm 0.07$ & $37.64 \pm 0.05$ & $37.42 \pm 0.07 *$ \\
\hline $25 a$ & $37.29 \pm 0.06$ & $38.32 \pm 0.07$ & $38.04 \pm 0.07$ & $37.34 \pm 0.09$ & $37.18 \pm 0.09$ \\
\hline $25 b$ & $37.35 \pm 0.05$ & $38.38 \pm 0.07$ & $37.85 \pm 0.08$ & $37.85 \pm 0.06$ & $37.46 \pm 0.09$ \\
\hline $25 \mathrm{c}$ & $37.44 \pm 0.08$ & $38.44 \pm 0.35$ & $37.92 \pm 0.07$ & $37.92 \pm 0.05$ & $37.42 \pm 0.08$ \\
\hline $\begin{array}{l}\text { Ref. Standard } \\
\text { (Acetaminophen) }\end{array}$ & $37.18 \pm 0.07$ & $37.88 \pm 0.05$ & $37.72 \pm 0.04$ & $37.41 \pm 0.04$ & $37.20 \pm 0.05^{* * *}$ \\
\hline
\end{tabular}

Note: The reaction time value is the mean $\pm \operatorname{SEM}(\mathrm{n}=6)$. Statistical analysis was performed with student's unpaired t-test (Kulkarni, 2003). ${ }^{*} \mathrm{p}<0.05,{ }^{* *} \mathrm{p}<0.01$ and ${ }^{* * *} \mathrm{p}<0.001,132 \mu \mathrm{mol} / \mathrm{kg}$ dose.

\section{CONCLUSIONS}

In this article, the synthesized pyrimidines 3, 7, 13a,b, 15, 17a,b, 21a,b, 25a, b, c; thiophenes 9a, b; coumarin 11, pyridine 19 and pyrroles $\mathbf{2 3} \mathbf{a}$, b were evaluated as analgesic, antipyretic and antiinflammatory agents compared to the reference standard drug acetaminophen. Among the newly synthesized compounds, compounds $\mathbf{1 1}$ and $\mathbf{1 9}$ showed promising significant analgesic and antipyretic activities compared to the other compounds. Additionally compounds 7 , 9a,b and 13a,b had moderately significant analgesic and antipyretic activities. Moreover, compound $\mathbf{1 1}$ had clear anti-inflammatory properties compared to the remaining compounds.

\section{ACKNOWLEDGEMENTS}

The authors would like to thank the research group working in the Pharamacology Depatment, Faculty of Pharmacy, October University for Modern Sciences and Arts. We would also like to thank the Poison Control and Medical Forensic Chemistry Center team from Jazan Health, Jazan City, Kingdom of Saudi Arabia for recording the analytical and spectral data for the newly synthesized compounds. 
TABLE III - Anti-inflammatory activities of the synthesized compounds

\begin{tabular}{|c|c|c|c|c|}
\hline \multirow{2}{*}{ Comp. No } & \multicolumn{2}{|c|}{ Change in reaction time (sec) \pm SEM } & \multicolumn{2}{|c|}{$\%$ Anti-inflammatory activity \pm SD } \\
\hline & $2 \mathrm{~h}$ & $4 \mathrm{~h}$ & $2 \mathrm{~h}$ & $4 \mathrm{~h}$ \\
\hline Control & $1.29 \pm 0.01$ & $1.32 \pm 0.007$ & & \\
\hline 3 & $0.83 \pm 0.01$ & $0.80 \pm 0.01$ & $34.56 \pm 3.38$ & $35.78 \pm 3.52$ \\
\hline 7 & $0.82 \pm 0.02$ & $0.83 \pm 0.01$ & $31.34 \pm 2.87$ & $33.59 \pm 2.26^{* *}$ \\
\hline $9 a$ & $0.82 \pm 0.01$ & $0.85 \pm 0.01$ & $32.44 \pm 2.55$ & $34.23 \pm 2.68$ \\
\hline $9 b$ & $0.81 \pm 0.03$ & $0.82 \pm 0.01$ & $31.12 \pm 3.51$ & $34.19 \pm 3.07 * *$ \\
\hline 11 & $0.82 \pm 0.02$ & $0.84 \pm 0.01$ & $29.34 \pm 2.97$ & $32.08 \pm 2.05 * * *$ \\
\hline $13 \mathbf{a}$ & $0.81 \pm 0.01$ & $0.85 \pm 0.01$ & $32.56 \pm 3.08$ & $33.38 \pm 3.17$ \\
\hline $13 b$ & $0.83 \pm 0.03$ & $0.84 \pm 0.01$ & $32.34 \pm 2.26$ & $35.27 \pm 2.02 * *$ \\
\hline 15 & $0.84 \pm 0.02$ & $0.81 \pm 0.01$ & $27.88 \pm 2.66$ & $32.17 \pm 1.87$ \\
\hline $17 \mathbf{a}$ & $0.83 \pm 0.02$ & $0.86 \pm 0.01$ & $34.23 \pm 3.44$ & $37.18 \pm 3.25$ \\
\hline $17 \mathrm{~b}$ & $0.86 \pm 0.01$ & $0.87 \pm 0.01$ & $35.56 \pm 3.14$ & $36.34 \pm 1.48$ \\
\hline 19 & $0.81 \pm 0.01$ & $0.83 \pm 0.01$ & $30.34 \pm 3.24$ & $34.03 \pm 2.53^{* *}$ \\
\hline $21 \mathrm{a}$ & $0.83 \pm 0.03$ & $0.85 \pm 0.01$ & $26.23 \pm 2.57$ & $29.28 \pm 1.38$ \\
\hline $21 b$ & $0.85 \pm 0.01$ & $0.81 \pm 0.01$ & $35.39 \pm 3.28$ & $37.31 \pm 1.93$ \\
\hline $23 a$ & $0.84 \pm 0.02$ & $0.88 \pm 0.01$ & $34.18 \pm 2.73$ & $35.98 \pm 3.02$ \\
\hline $23 b$ & $0.78 \pm 0.02$ & $0.82 \pm 0.01$ & $33.85 \pm 3.27$ & $35.58 \pm 2.17$ \\
\hline $25 a$ & $0.83 \pm 0.02$ & $0.80 \pm 0.01$ & $33.37 \pm 3.26$ & $36.18 \pm 1.59$ \\
\hline $25 b$ & $0.79 \pm 0.01$ & $0.83 \pm 0.01$ & $32.94 \pm 2.62$ & $35.42 \pm 3.46$ \\
\hline $25 \mathrm{c}$ & $0.83 \pm 0.03$ & $0.87 \pm 0.01$ & $34.23 \pm 3.17$ & $36.14 \pm 1.83$ \\
\hline $\begin{array}{l}\text { Ref. Standard } \\
\text { (Acetaminophen) }\end{array}$ & $0.83 \pm 0.01$ & $0.84 \pm 0.01$ & $28.83 \pm 3.05$ & $32.82 \pm 1.33 * * *$ \\
\hline
\end{tabular}

Note: The reaction time value is the mean $\pm \operatorname{SEM}(n=6)$. Statistical analysis was performed with student's unpaired t-test (Kulkarni, 2003). ${ }^{*} \mathrm{p}<0.05, * * \mathrm{p}<0.01$ and $* * * \mathrm{p}<0.001,132 \mu \mathrm{mol} / \mathrm{kg}$ dose.

\section{REFERNCES}

Agarwal A, Ashutosh R, Goyal N, Chawhan PMS, Gupta S. Dihydropyrido $[2,3-d]$ pyrimidines as a new class of antileishmanial agents. Bioorg Med Chem. 2005;13(24):66786684.

Dallemagne P, Khanh LP, Alsaidi A, Varlet I, Collot V, Paillet M, Bureau R, Rault S. Synthesis and biological evaluation of fiveMembered heterocycles fused to cyclopenta[ $[$ ] thiophene as new antitumor agents. Bioorg Med Chem. 2003;11(7):1161-1167.

El-Sharkawy KA, Nasser NE, Zaki MY. Uses of 2-Amino-5, 6-dihydro- $4 H$-cyclopenta $[b]$ thiophene-3-carbonitrile in the synthesis of novel heterocyclic compounds with anticonvulsant, behavioral and CNS antidepressant activities. Int Res J Pure App Chem. 2012;2(1):91-104.
Fadeyi OO, Obafemi CA, Adewunmi CO, Iwalewa EO. Antipyretic, analgesic, anti-inflammatory and cytotoxic effects of four derivatives of salicylic acid and anthranilic acid in mice and rats. Afr J Biotech. 2004;3(8):426-431.

Gus 'Kov EP, Prokofev VN, Kletskii ME, Kornienko IV, Ogapurenko A, Olekhnovich LP, Chistyakov VA, Shestopalov AV, Sazykina MA. Allantoin as a vitamin. Dokl Biochem Biophys. 2004;398:320-324.

Horvati K, Bacasa B, Szabo N, Fodor K, Balaka G, Rusavai M, Kiss É, Mezo G, Grolmusz V, Vertessy B, Hudecz F, Bosze S. Antimycobacterial activity of peptide conjugate of pyridopyrimidine derivative against Mycobacterium tuberculosis in a series of in vitro and in vivo models. Tuberculosis. 2015;95(Suppl 1):S207-S211. 
Iqbal Z, Hameed S, Ali S, Tehseen Y, Shahid M, Iqbal J. Synthesis, characterization, hypoglycemic and aldose reductase inhibition activity of arylsulfonylspiro[fluorene-9, $5^{\prime}$ imidazolidine]-2',4'-diones. Eur J Med Chem. 2015;98:127-138.

Keri RS, Hosamani KM, Shingalapure RV, Hugar MH. Analgesic, anti-pyretic and DNA cleavage studies of novel pyrimidine derivatives of coumarin moiety. Eur J Med Chem. 2010;45(6):2597-2605.

Kulkarni SK. Handbook of Experimental Pharmacology. 3 ed. Delhi: Vallabh Prakashan; 2003. P. 123-125; 128-131; 172-186.

Mohareb R, El-Sharkawy KA, Sherif SM. The reaction of $\beta$-amino- $\alpha, \gamma$-dicyano -crotononitrile with acetophenone: Synthesis of pyridine, pyridazine and thiophene derivatives with antimicrobial activities. Acta Pharm. 2008;58:429-444.

Padmavathi V, Prema Kumari C, Venkatesh BC, Padmaja A. Synthesis and antimicrobial activity of amido linked pyrrolyl and pyrazolyl-oxazoles, thiazoles and imidazoles. Eur J Med Chem. 2011;46(11):5317-5326.

Rajesh SM, Kumar RS, Libertsen LA, Perumal S, Yogeeswari P, Sriram D. A green expedient synthesis of pyridopyrimidine2-thiones and their antitubercular activity. Bioorg Med Chem Lett. 2011;21(10):3012-3016.
Reddy YT, Reddy PN, Kodru S, Damodran C, Crooks PA. Aplysinopsin analogs: Synthesis and anti-proliferative activity of substituted (Z)-5-(N-benzylindol-3-ylmethylene) imidazolidine2,4-diones. Bioorg Med Chem. 2010;18(10):3570-3574.

Sztanke M, Rzymowska J, Sztanke K. Synthesis, structure elucidation and in vitro anticancer activities of novel derivatives of diethyl (2E)-2-[(2E)-(1-aryl- imidazolidin-2-ylidene) hydrazono]succinate and ethyl (4-oxo-8-aryl-4,6,7,8-tetrahydroimidazo[2,1-c][1,2,4]triazin-3-yl)acetate. Bioorg Med Chem. 2013;21(23):7465-7480.

Vogel HG. Drug discovery and evaluation. Pharmacological assay. 2 ed. Berlin, Heidelberg: Springer-Verlag; 2002. p. 695$696 ; 759-762 ; 772-773$.

Wang G, Wang Y, Wang L, Han L, Hou X, Fu H, Fang H. Design, synthesis and preliminary bioactivity studies of imidazolidine2,4-dione derivatives as Bcl-2 inhibitors. Bioorg Med Chem. 2015;23(23):7359-7365.

Received for publication on $16^{\text {th }}$ October 2016 Accepted for publication on $09^{\text {th }}$ January 2018 